\title{
SOBRE EL CARÁCTER SECUNDARIO Y DISCRECIONAL DEL REMEDIO DEL CUMPLIMIENTO ESPECÍFICO EN EL COMMON LAW. PERSPECTIVA HISTÓRICA Y APROXIMACIÓN ACTUAL
}

\author{
SPECIFIC PERFORMANCE IN COMMON LAW: AN EQUITABLE AND \\ SECONDARY REMEDY. HISTORICAL AND CURRENT PERSPECTIVES
}

\author{
GonZalo SeVerin Fuster $^{* *}$
}

\begin{abstract}
RESUMEN: El objeto de este trabajo es dar cuenta de la aproximación del Common Law al remedio del cumplimiento específico del contrato, a fin de determinar hasta qué punto puede tenerse por efectiva la idea generalizada de que, frente al incumplimiento contractual, el acreedor no tiene derecho al cumplimiento específico, y que el remedio primario o preferente es la indemnización de daños. Después de mostrar que esta afirmación de entrada parece tener suficiente respaldo, y tras delimitar su alcance desde una perspectiva terminológica, el resto del trabajo ahonda sobre esa preferencia por el remedio indemnizatorio: cuál es su explicación histórica y cuál es su significación en la actualidad.
\end{abstract}

Palabras clave: Incumplimiento contractual, Remedios, Cumplimiento específico, Indemnización de daños, Common Law.

ABSTRACT: This paper deals with specific performance in the Common Law. The main purpose here is to determine whether the widespread idea that Common Law dismisses specific performance as a remedy in case of breach of contract is effective. The work studies why there is a trend in this legal tradition to award damages over specific performance in cases of contractual breach. This issue is undertaken from a historical and current perspective.

Key words: Breach of contract, Remedies, Specific performance, Damages, Common Law.

\section{INTRODUCCIÓN}

Destacar el diferente lugar que ocupa el remedio de cumplimiento específico - "specific performance"- dentro del sistema de remedios por el incumplimiento contractual en el Common Law y en el Derecho continental, es casi un lugar común en los trabajos de Derecho comparado. En este sentido, se afirma que mientras que en el Derecho continental se reconoce al acreedor un derecho a obtener el cumplimiento específico, en el Common Law

\footnotetext{
Profesor de Derecho civil de la Facultad de Derecho de la Pontificia Universidad Católica de Valparaíso, Chile. Dirección postal: Avenida Brasil 2950, Valparaíso. Dirección electrónica: gonzalo.severin@ucv.cl.

Este trabajo es una adaptación del capítulo sexto de la tesis doctoral inédita titulada "Los contratos de servicio: su construcción como categoría contractual, y el derecho del cliente al cumplimiento específico”, defendida en Madrid el 20 de junio del año 2014. Agradezco a mi directora, la profesora Nieves Fenoy Picón, todas las sugerencias y los comentarios realizados en su oportunidad. Los errores son, lógicamente, de mi exclusiva responsabilidad.
} 
este remedio se considera un remedio secundario, que se concede solo excepcionalmente, siendo la indemnización de los daños - “damages"- el remedio preferente1.

Los mismos estudios comparados sugieren, sin embargo, que dicha diferencia es más teórica que práctica ${ }^{2}$. Lógicamente, es posible pensar que ello puede deberse tanto al hecho de que los ordenamientos de Derecho continental son, en la práctica, más restrictivos a la hora de conceder el cumplimiento específico de lo que generalmente se cree, como al hecho de que el remedio del specific performance ocupa un lugar más relevante en el Common Law de lo que suele afirmarse. En este trabajo interesa analizar en qué medida la afirmación sobre el lugar secundario que tiene el remedio del cumplimiento específico en el Common Law puede tenerse por cierta; y en su caso, cuál es la justificación y el alcance de esa aproximación; tomando como referencia al Derecho inglés, si bien al hilo de la exposición se hacen algunas referencias a otros ordenamientos pertenecientes a esa familia jurídica.

La exposición que sigue se estructura en tres partes. En la primera parte se contemplan unas consideraciones preliminares. Son dos las cuestiones que, creo, conviene tratar antes de comenzar el estudio. Una, consiste en determinar si dicha afirmación de entrada -el lugar secundario del remedio del specific performance- coincide con la forma en que la propia doctrina del Common Law describe, en general, el sistema de remedios frente al incumplimiento contractual. La otra, consiste en realizar algunas precisiones terminológicas, considerando que se ha sugerido que el diferente significado que se atribuye a la expresión specific performance y a la del cumplimiento específico en los distintos ordenamientos puede incidir en que las diferencias entre el Common Law y el Derecho continental "sean menos serias de lo que a primera vista aparecen"3.

Partiendo de la base de que la afirmación sobre el lugar secundario del remedio del specific performance parece tener suficiente respaldo, y habiendo delimitado su alcance desde una perspectiva terminológica, el resto del trabajo pretende ahondar sobre el alcance de esa "preferencia". Por ello, la segunda parte de este trabajo muestra cuál es la explicación histórica de la preferencia por el remedio de los damages sobre el del specific performance. Y la tercera parte versa sobre cuál es su significación en la actualidad, mostrando cuáles son los principales criterios que están a la base de la decisión de otorgar o no este remedio. Al final se recogen las principales conclusiones.

\footnotetext{
1 Así, por ejemplo: Beale et al. (2010) p. 834; Cartwright (2007) p. 248; De Cruz (1999) p. 398; HogG (2011) p. 349; MARKESINIS et al. (2006) p. 263 y pp. 392 y ss. (comparando el Derecho inglés con el Derecho alemán); Miller (2009) p. 145 (comparando el Derecho inglés con el Derecho francés); Torsello (2006) pp. 617 y ss.; Youngs (1998) pp. 434 y ss. (comparando el Derecho inglés con el francés y con el alemán); ZIMMERMANN (1992) pp. 776 y ss.; ZweIGERT y KÖTZ (1998) pp. 470-485.

2 Así, por ejemplo, ZwEIGERT y KÖTZ (1998) p. 484, afirman: "the actual contrast is not quite sharp". Y en el mismo sentido, BEALE et al. (2010) p. 854: "the differences between the system may be less serious than appears at first sight [...] it is thought that practice, at least in Germany, reduces the differences still further". Véase, igualmente, la exposición que, sobre este punto, se ofrece en FAUVArQUE-Cosson y MAZEAUd (2008) pp. 479-480 (con comentarios al Derecho francés, alemán y suizo). Desde luego, esa idea no es nueva. Ya TREITEL (1976) p. 8, en un conocido trabajo de Derecho comparado sobre los remedios por incumplimiento, ponía de relieve que "the availability of enforced performance is in fact limited in all the legal systems under discussion". 3 Cfr. Beale et al. (2010) p. 854.
} 


\section{CONSIDERACIONES PRELIMINARES}

\subsection{LA AFIRMACIÓN DE ENTRADA}

Una mirada a la doctrina del Common Law demuestra que la afirmación de que el specific performance es un remedio secundario tiene suficiente respaldo. Si tomamos como punto de partida al Derecho inglés, las palabras de BuRrows son elocuentes: "Another question [...] is whether a plaintiff has a free choice between claiming specific enforcement or compensation. In English law the answer is "no». The enforcement of positive obligations (other than payments already owed) through the remedy of specific performance is secondary to compensation; i.e. a plaintiff is only entitled to specific performance [...] where compensatory damages would be inadequate" ${ }^{4}$.

Y con igual contundencia, se pronuncia, por ejemplo, STONE, quien afirma: "Since the remedy [of specific performance] is an equitable one [...] it is discretionary, unlike damages, which are available as of right. This means that a claimant is not entitled to the order simply as a result of proving that the other party is in breach of its obligations" 5 .

Esas descripciones sobre el remedio del specific performance no solo son ampliamente compartidas por otras autorizadas voces de la doctrina inglesa ${ }^{6}$, sino que también lo son por los tribunales. Las palabras de Lord Hoffman [Co-operative Insurance Society Ltd v. Argyll Stores (1997) UKHL 17] sintetizan con claridad la aproximación tradicional del Derecho inglés al specific performance: "Specific performance is traditionally regarded in English law as an exceptional remedy, as opposed to the common law damages to which a successful plaintiff is entitled as of right [...] specific performance will not be ordered when damages are an adequate remedy"

La descripción de la forma en que opera el sistema de remedios frente al incumplimiento contractual es similar en el Derecho estadounidense. Así, por ejemplo, FARNSWORTH sostiene: "[A]lthough the injured party can always claim damages for breach of contract, that party's right to specific relief as an alternative is much more limited [...] the limits on specific relief [...] are peculiar to the common law and are unknown in other legal systems" ${ }^{\prime}$.

\footnotetext{
${ }^{4}$ Burrows (1998) p. 137.

5 STONE (2009) p. 632.

${ }^{6}$ Así, por ejemplo: Andrews (2011) pp. 530 y ss.; Cartwright (2007) pp. 247 y ss.; Furmston (2007) pp. 798-799; Samuel (2001) pp. 158 y ss.; Smith y Atiyah (2005) pp. 377-378; y Treitel (2003) pp. 1019 y ss. El lugar secundario del remedio de specific performance explica el limitado tratamiento que se le da a este remedio en los manuales de Derecho contractual inglés, en comparación con el que se da al remedio de los damages [c $f$ r. por ejemplo, CHARMAN (2007), quien únicamente enumera el remedio de cumplimiento específico bajo el epígrafe "otros remedios", para el caso en que el remedio de daños no sea "justo" (pp. 229-230); Mulcahy (2008) quien apenas dedica una página a la descripción de este remedio (p. 209); o TuRNER (2007), quien le dedica apenas un par de páginas (pp. 214-215)].

7 Este caso, relativamente reciente, aparece citado con frecuencia por la doctrina inglesa. Vuelvo sobre él un poco más adelante.

8 FANSWORTh (2010) p. 162.
} 
Se trata, esta, de una opinión compartida por la mayoría de los autores estadounidenses $^{9}$, y de hecho, ha sido recogida en el Rest. $\left(2^{\text {nd }}\right)$ CONTRACTS $^{10}$. Es más, es precisamente en la doctrina estadounidense en donde se puede hallar la afirmación más categórica en relación con el lugar secundario del remedio del cumplimiento específico en el Common Law. La hizo el juez O. W. Holmes en 1882, quien afirmó: "The remedy [of specific performance] is an exceptional one. The only universal consequence of a legally binding promise is, that the law makes the promisor pay damages if the promised event does not come to pass. In every case it leaves him free from interference until the time for fulfilment has gone by, and therefore free to break his contract if he chooses"11. Y en otro lugar, Holmes afirmó: "The duty to keep a contract in common law means a prediction that you must pay damages if you do not keep it, and nothing else"12. La interpretación de esta última afirmación de Holmes como descriptiva de la preferencia por el remedio de daños en el Common Law (esto es, que el giro "and nothing else" significaría "no existe otro remedio") es la más usual, y en este sentido, es todavía comúnmente citada ${ }^{13}$. Conviene destacar que la idea que se desprende de ello -la de concebir el contrato como una opción entre "cumplir o pagar" - ha sido recogida por la doctrina del análisis económico del Derecho para sugerir que, en la medida que tal opción está determinada por la búsqueda de la eficiencia, la preferencia por el remedio de los damages implica que el ordenamiento jurídico promueve -correctamente- el incumplimiento del contrato en ciertos $\operatorname{casos}^{14}$. Por otro lado, hay que destacar que aunque la doctrina inglesa estima que la apreciación de Holmes puede parecer exagerada, se admite que da cuenta de cómo funciona el sistema de remedios en la práctica ${ }^{15}$.

\footnotetext{
9 "It is generally recognized among contract scholars that the injured party's expectation interest can be protected by either money damages or injunctive relief [...] [m]ost [scholars] also agree, however, that U.S contract law exhibits a strong preference for money damages, and the injunctive relief is available only in exceptional circumstances" KLASS (2010) p. 196.

10 REST (2 ${ }^{\mathrm{ND}}$ ) CONTRACTS $₫ 359$ (Effect of Adequacy of Damages): "(1) Specific performance or an injunction will not be ordered if damages would be adequate to protect the expectation interest of the injured party".

11 Holmes (1882) pp. 300-301.

12 Holmes (1998) p. 702.

13 Sin embargo, esta interpretación de las palabras de Holmes ha sido criticada, sosteniéndose que no se trata de una afirmación relativa al Derecho contractual, sino a la naturaleza del "deber" en sentido jurídico, y además, limitada al Common Law en sentido estricto (esto es, sin considerar la Equity). Desde esta perspectiva, la expresión "and nothing else", contenida en la afirmación de Holmes, significaría que la noción de deber no tiene ninguna otra connotación moral, teológica o de cualquier otro tipo [cfr. SMITH (2005) pp. 222-224].

${ }^{14}$ La potencial justificación de la preferencia por el remedio de daños (damages) sobre la base de los postulados del análisis económico del Derecho no es objeto de este estudio. Sin perjuicio, para una aproximación a ello, véase, por ejemplo, Kronman (1978) y Shavell (2006). En todo caso, sabe destacar que el valor descriptivo de la afirmación de Holmes, en el sentido de que el Common Law no reconoce un derecho al cumplimiento específico, no se pone en tela de juicio ni siquiera por quienes están a favor de que el cumplimiento específico tenga un lugar más relevante, o que deba incluso ser considerado el remedio preferente [por ejemplo: SCHWARTZ (1980)].

${ }^{15}$ Así, por ejemplo, CARTWRIGHT expresa: "[t]he English lawyer would not generally make such as strong statement. The primary obligation is seen as the obligation to perform"; pero agrega inmediatamente: "The way in wich the remedies for breach of contract operate does in practice translate non-performance or defective performance into money-damages. The courts are reluctant to order a party to perform, and therefore the party in breach can in most cases pay damages rather than performing his obligations if he chooses" [(2007) p. 248]. En un sentido parecido, Smith y AтiYaH (2005) pp. 372-373, también a partir de los postulados de Holmes.
} 
La preferencia por el remedio de los damages y el lugar secundario del specific performance se describe, en los mismos términos en los que se ha expresado arriba, en otros ordenamientos del Common Law, como por ejemplo, el australiano ${ }^{16}$, el canadiense ${ }^{17}$, y el neozelandés ${ }^{18}$.

Lo dicho hasta ahora permite tener por cierta la afirmación de entrada: en el Common Law, el remedio del specific performance es un remedio "secundario" frente al remedio de los damages. Y la percepción de que ello contrasta con el reconocimiento del derecho del acreedor a obtener el cumplimiento específico en los ordenamientos de Derecho continental, es también una idea compartida por la doctrina jurídica del Common Law, aunque se sugiere que, posiblemente, la diferencia en la práctica no sea tan tajante ${ }^{19}$.

\subsection{Algunas PRECisiones TERMinOlÓgICAS}

\section{A. La expresión "specific performance"}

En el Common Law, el remedio del specific performance consiste en una orden del tribunal dirigida a la parte que ha incumplido el contrato, para que ejecute la obligación personalmente en los términos pactados ${ }^{20}$, bajo la amenaza del uso de vías de coerción para el caso que no cumpla con lo ordenado ${ }^{21}$.

Sin embargo, la expresión "specific performance" admite dos acepciones. En un sentido amplio, "specific performance" se refiere a cualquier obligación, ya sea positiva o negativa, caso en el cual es sinónima de "specific relief"22. En un sentido restringido, el remedio del "specific performance" únicamente se refiere a las obligaciones positivas (a las de dar y a las de hacer), distinguiéndose de la vía o el remedio para obtener el cumplimiento de una obligación negativa (las de no hacer), que se denomina injunction.

16 Cfr. Carter et al. (2007) pp. 937-941; Monahan (2001) p. 160. En el mismo sentido, Musgrave (2009) pp. 300-372, ofreciendo una comparación de los remedios entre el Derecho australiano y el Derecho francés.

17 Cfr. JUKier (2010) pp. 85-118; y STACK (2000) p. 62.

18 Cfr. Cuncannon (2004) pp. 657-658.

19 En este sentido, Burrows afirma que la preferencia por el remedio de daños en el Derecho inglés "contrasts with the position in civilian jurisdictions [...] where specific implement is the primary remedy", aunque agrega inmediatamente: "although I suspect that the difference is not as clear-cut as it is often alleged to be" [(1998) p. 137]. En términos muy similares, Lord Hoffman [Co-operative Insurance Society Ltd v. Argyll Stores (1997) UKHL 17]: "Specific performance is traditionally regarded in English law as an exceptional remedy [...] [b]y contrast, in countries with legal systems based on civil law, such as France, Germany and Scotland, the plaintiff is prima facie entitled to specific performance. The cases in which he is confined to a claim for damages are regarded as the exceptions [...] [i]n practice, however, there is less difference between common law and civilian systems than these general statements might lead one to suppose".

En la doctrina estadounidense, en términos parecidos, FARnswORTH: "[ $\mathrm{t}$ ]his strong preference [...] stands in sharp contrast to the preference of civil law system, those derived from the Roman Law, for specific performance" [(2010) p. 164].

20 Cfr. Cartwright (2007) p. 251; también Fry (1911) p. 2 (en relación con el Derecho inglés); y FarnsworTH (2010) p. 167, y KLASS (2010) p. 211 (en relación con el Derecho estadounidense).

21 A la parte que ha incumplido se le puede considerar culpable de desacato al tribunal (contemnor), y en consecuencia, arriesga diferentes posibles sanciones: prisión, pago de multas, y secuestro o embargo de bienes, en su caso, seguido de su venta [cfr. ANDrews (2011) p. 530].

22 El Black's Law Dictionary (2009) remite, en la voz o entrada "specific relief”, a "specific performance". La voz "relief” significa lo mismo que "remedy", aunque aquella aparece más comúnmente cuando se trata de remedios “equitativos" [cfr. LANGDELL (1908) p. 19]. 
La injunction es una orden dirigida a la parte incumplidora para que realice un determinado acto (por ejemplo, que destruya un muro que ha construido en contravención del contrato) o para que se abstenga de realizar un determinado acto (por ejemplo, celebrar o ejecutar un segundo contrato que implicaría incumplir el primero). La primera clase se denomina "mandatory injuction" y la segunda "prohibitory injunction"23. Si bien la injunction no es propiamente una vía para obtener la ejecución forzosa de una obligación de hacer, puede, en ciertos casos, ser una vía indirecta para promover su cumplimiento ${ }^{24}$.

La distinción entre el remedio del specific performance y la injunction puede ser bien ejemplificada con el conocido caso Lumley v. Wagner [(1852) EWHC Ch J96]: una cantante de ópera se había comprometido a realizar una serie de representaciones en un teatro, en exclusiva, por un tiempo determinado. Sin embargo, la cantante celebra otro contrato para cantar en un teatro distinto durante el mismo período. En el caso, el tribunal no ordena a la cantante cumplir con su obligación de cantar derivada del primer contrato (specific performance), sino que le prohíbe cumplir con el segundo contrato (injunction) ${ }^{25}$.

La precisión sobre el significado de la expresión specific performance resulta importante, sobre todo desde la perspectiva de un estudio comparado. En el Derecho continental, expresiones tales como "cumplimiento específico", "ejecución in natura" y "cumplimiento forzoso de forma específica", se refieren tanto a las obligaciones positivas (dar y hacer) como a las negativas (no hacer). Y además -y esto es más relevante- hay que considerar que, como remedio frente al incumplimiento, dichas expresiones son utilizadas para indicar el hecho de que el acreedor recibe en especie la prestación debida, no únicamente cuando la ejecuta el propio del deudor, sino también cuando se obtiene a través de la actuación del tribunal o incluso, de un tercero ${ }^{26}$.

23 Cfr. Cartwright (2007) p. 251; y Furmston (2007) pp. 800 y ss. Como se puede ver, la injunction, en cuanto tiende a obtener la satisfacción del acreedor en los términos originalmente pactados, es también una vía de "cumplimiento específico", lo que explica que, en sentido amplio, la expresión specific performance abarque este remedio cfr. OUGHTON y Davis (2000) p. 519. La voz "specific performance", en consecuencia, no permite distinguir este remedio (en sentido restringido) de otros remedios equitativos como la injunction. La idea de "specific" más bien destaca la diferencia que existe entre esta vía y la vía alternativa de la indemnización de daños, que es un remedio de cumplimiento "por equivalente" cfr. FRY (1911) p. 2.

24 En efecto, si por alguna razón el cumplimiento específico de la obligación no puede ser directamente ordenado, la corte puede emitir una negative injunction, ordenando que la parte incumplidora evite ejecutar actos inconsistentes con el cumplimiento $c f r$. KLASS (2010) p. 211; FARnSWORTH (2010) p. 168. Sin embargo, esta posibilidad está limitada a otras reglas, como por ejemplo, que ello no tenga por efecto obligar a una persona a ejecutar un servicio personal $c f r$. ANDrews (2011) p. 520. Sobre la imposibilidad de obtener el cumplimiento específico en los contratos de "servicios personales", trato más adelante.

25 Otro famoso caso es Warner Bros v. Nelson [(1937) 1 KB 209]. La actriz Bette Davis había firmado un contrato en exclusiva con Warner Bros., contrato que la actriz incumplió, celebrando otro contrato para filmar una película para una compañía inglesa. También en este caso se concedió una injunction, prohibiendo a la actriz cumplir con el segundo contrato. En este caso, al igual que en Lumley v. Wagner, existía una cláusula expresa de exclusividad (por tanto, una obligación contractual de no hacer). Un análisis de estos dos casos aquí citados, y de otros similares, en TANnENBAUM (1954) pp. 18-27.

26 Cfr. Beale et al. (2010) p. 840; y también Romero (1986) pp. 788-789, con especial referencia al Derecho francés y al de Quebec. En la doctrina española, EsTeban y Gómez (2013) p. 1600, explican que es importante diferenciar la expresión "cumplimiento específico" (término que -expresan- deriva del specific performance del Common Law y que pone el acento en la posibilidad de ordenar al demandado que cumpla) de la expresión 


\section{B. El TÉRMINO “DAMAGES"}

En el contexto del incumplimiento contractual, el remedio de los damages (literalmente "daños") se refiere a la compensación monetaria que el tribunal otorga a la parte no incumplidora. Es, por oposición al specific reliefo specific performance en sentido amplio, un substitutionary relief ${ }^{27}$.

Lo que se busca con este remedio es dejar a la parte no incumplidora en la situación en la que se encontraría de haberse cumplido el contrato ${ }^{28}$. La medida de estos daños se calcula sobre la base del beneficio que el contrato le confería a la parte - "loss of bargain"-, aunque no se trata de todo potencial beneficio, sino únicamente de aquello que, en virtud del contrato, el acreedor podía razonablemente esperar. De ahí que a la medida de estos daños se les denomine "expectations damages"29. Los expectations damages se distinguen de otros daños que, si bien se conceden excepcionalmente en caso de incumplimiento contractual, no están orientados a proteger el interés del acreedor en el cumplimiento del contrato, sino que buscan dejar al acreedor en la misma posición en la que se encontraría de no haberse celebrado el contrato. Estos se denominan "reliance damages" 30 . Por lo dicho, si en el estudio de la preferencia del remedio de damages sobre el specific performance se traduce "damages" por "indemnización de perjuicios", hay que considerar que con ello se está

\footnotetext{
"cumplimiento in natura", que sería la denominación más adecuada para referirse a los sistemas de Derecho continental. Destacan estas autoras que en el Derecho continental suele considerarse que existe ejecución in natura en la medida que el acreedor obtiene en especie la promesa contractual, aunque no sea del demandado, "sino también por un tercero, por un oficial de justicia o, incluso, por el propio demandante a expensas del demandado (deudor)". En el mismo sentido, Morales (2006) p. 89: "[s]on medidas de cumplimiento todas las que se dirigen a procurar la satisfacción del acreedor ejecutante, de modo directo o indirecto", incluyendo entre ellas no solo "las orientadas a lograr el cumplimiento del deudor en los propios términos", sino también "las que se dirigen a lograr la satisfacción in natura del acreedor ejecutante, sustituyendo la actividad del deudor por la del juez o la de otro sujeto, con cargo al deudor". Sobre la explicación histórica de esta noción de specific performance en el Common Law, entendida como una orden dirigida al deudor, trato un poco más adelante.

27 En efecto, se trata de daños “in substitution of”, es decir, daños en sustitución o en lugar del cumplimiento [s. 50 Senior Court Act 1981 de UK (Power to award damages as well as, or in substitution for, injunction or specific performance): "Where the Court of Appeal or the High Court has jurisdiction to entertain an application for an injunction or specific performance, it may award damages in addition to, or in substitution for, an injunction or specific performance"]. La doctrina utiliza también la expresión "damages in lieu of specific performance" [ $v$. gr. Samuel (2001) p. 146]. Es posible, lógicamente, que se ordene el cumplimiento específico del contrato y además una indemnización de daños, ya que la ejecución in natura, aunque sea posible, no satisfará al acreedor, porque la prestación no se obtiene en el momento en que era debida [cfr. LANDGELL (1908) p. 43].

28 Las palabras de Lord Nicholls of Birkenhead [Attorney General v. Blake and Another (2000) UKHL 45] ilustran con claridad el punto: "[t] he basic remedy is an award of damages [...] the rule of the common law is that where a party sustains a loss by reason of a breach of contract, he is, so far as money can do it, to be placed in the same position as if the contract had been performed [...] Leaving aside the anomalous exception of punitive damages, damages are compensatory. That is axiomatic".

29 La denominación de estos intereses fue acuñada en la doctrina estadounidense [se debe al conocido trabajo "The reliance interest in contract damages", de Fuller y Perdue (1936)]. Sin embargo, es terminología utilizada también por la doctrina inglesa $v$ gr. SAMUel (2001) pp. 195-203; CARTWRight (2007) p. 262; STONE (2009) p. 596; ANDREWs (2011) p. 519.

30 Sobre la posibilidad de reclamar los reliance damages, por incumplimiento contractual, RICHARDS (2009) p. 388, explica: "[t]he circumstances in which a party claims reliance loss may arise where the profits that they hope will materialise from the contract are too speculative or uncertain. In such circumstances the injured party may find it simpler and safer to claim any expenses incurred in performance of the contract".
} 
haciendo generalmente referencia al cumplimiento por equivalente de la prestación, y no a toda pretensión indemnizatoria que pueda tener base en el incumplimiento del contrato. Esto se relaciona estrechamente con el concepto más o menos amplio que se tenga del "incumplimiento contractual”, como se verá a continuación.

\section{LA NOCIÓN DE “BREACH OF CONTRACT"}

En el Common Law, tanto el remedio del specific performance como el remedio de los damages operan en el caso que exista un breach of contract. La noción de breach of contract incluye tanto la falta de ejecución como los supuestos de ejecución defectuosa o tardía. Sin embargo, no comprende los supuestos en los que, como consecuencia de eventos imprevistos y fuera del control de las partes, se ha frustrado el propósito del contrato, o su cumplimiento se ha vuelto imposible, o su cumplimiento implicaría un cambio radical en los términos originales del contrato. En estos casos, el deudor queda liberado de su obligación, y en consecuencia, no puede contarse con el remedio del specific performace ni con el remedio de los damages. Dicho de otro modo, el deudor no es responsable del incumplimiento ${ }^{31}$. Ahora bien, esto no quiere decir que el acreedor no pueda recuperar el precio (o la parte del precio) que ha pagado; lo que ocurre es que tal pretensión restitutoria no se considera un remedio frente al incumplimiento (breach of contract), sino una aplicación del principio del enriquecimiento injustificado, que en el Common Law no se trata a propósito del Contract Law, sino del Law of restitution ${ }^{32}$.

31 Para una presentación de estos supuestos, tratados bajo la denominación de frustration, de imposibility y de impracticability, véase, en relación con el Derecho inglés, CARTWRIGHT (2007) pp. 235 y ss. [y considérese que algunos aspectos relativos a sus efectos han sido objeto de regulación en la Law Reform (frustrated contracts) Act 1943]; y en relación con el Derecho estadounidense, KLASS (2010) pp. 184 y ss. [y considérese que la doctrina se encuentra recogida en el Rest (2ND) CONTRACTs, $\$ 261$ - \$272 y en el UCC s. 2-615]. Por último, para un estudio de Derecho comparado sobre el efecto del cambio de circunstancias en la posibilidad de exigir el cumplimiento de los contratos, véase Momberg (2011) pp. 139 y ss.]. Por otro lado, hay que tener presente que la distinción entre breach of contract y frustration no siempre es clara, porque, en definitiva, se trata de una cuestión de interpretación del contrato: supone determinar si puede decirse o no que el deudor haya asumido el riesgo de que suceda dicho evento. En este sentido, las palabras de Sмiтн у АтіYан (2005) p. 186, son elocuentes: "Frustration must obviously be distinguished from breach of contract: if the performance of the contract is rendered impossible by the fault of either party, that party will be guilty of breach of contract and have to pay damages to the other party [...] [b] ut there are cases in wich it is difficult to draw the line between genuine frustration and breach because it is not clear what meaning is to be given to "fault" in this connection". En todo caso, la doctrina de la frustration es de aplicación muy restrictiva, de modo que, en principio, la falta de ejecución del contrato se mira como breach of contract [cfr. CARTWRIGHT (2007) pp. 239-241].

32 Estas pretensiones restitutorias se encausan en una demanda de daños, y por tanto, ellas configuran una tercera categoría de daños, frente a las otras dos ya mencionadas (expectations damages y reliance damages): los restitutionary damages. Los restitutionary damages deben, por tanto, distinguirse de los anteriores tipos de daños. RICHARDS (2009) pp. 389-390, lo explica en estos términos: "it is also possible to claim for the recovery of a benefit received by the defendant from the unperformed contract. Such claims are based in restitution [...] [c]laims in restitution are conceptually different from those for damages for breach of contract [se refiere a los expectations damages] since the aim is to place both the parties in the same position that they were in before the contract had been entered into, that is, as if the contract had not been made. It should be noted that restitution is not the same as claims for reliance loss either since, while the intention in the latter is to compensate for losses sustained before the contract was entered into, it is only intended to return the plaintiff to the pre-contract position and not the defendant, and the latter may be left in a significantly worse position than before". 
Así, el concepto de breach of contract, aun cuando pueda traducirse como "incumplimiento del contrato", no se corresponde con la moderna noción de "incumplimiento de contrato" que viene proponiendo un sector de la doctrina ${ }^{33}$ : si bien el breach of contract es una noción "amplia" (en el sentido que engloba tanto la falta de ejecución, como los supuestos de ejecución imperfecta y tardía), no parece que sea un concepto "neutro" desde el punto de vista de la imputación subjetiva al deudor.

\section{LA PREFERENCIA POR EL REMEDIO DE LOS DAMAGES Y EL CARÁCTER SECUNDARIO Y DISCRESIONAL DEL SPECIFIC PERFORMANCE. UNA BREVE APROXIMACIÓN HISTÓRICA}

El lugar secundario que se atribuye al remedio del specific performance frente al remedio de los damages se explica, en buena medida, porque aquel es un remedio "equitable" 34 . Con ello se está haciendo referencia a su origen histórico: el specific performance es un remedio creado por la Equity. La exposición que sigue tiene por objeto dar cuenta de este origen histórico ${ }^{35}$.

\subsection{EL COMMON LAW Y EL SURGIMIENTO DE LA EQUITY}

Como es sabido, el origen del Common Law inglés se sitúa temporalmente en la segunda mitad del siglo XI, tras la conquista normanda (1066), con el surgimiento de las cortes reales, las king's courts ${ }^{36}$.

Tras la conquista normanda, se pone en marcha un proceso de unificación y centralización territorial, que lógicamente también incide en el Derecho y en la forma de administración de Justicia. El origen de las king’s courts se sitúa en este contexto: los primeros jueces son miembros del Consejo real, que viajaban a través del país, revisando la actuación de las cortes locales. Las king's courts, una vez establecidas como tribunales autónomos, buscaron, con el fin de resolver con criterios similares en todo el territorio, lo que había "de común" en el Derecho consuetudinario aplicado por las distintas cortes locales. De ahí la deno-

33 Sobre esta moderna noción de incumplimiento - "amplia” y "neutra"- véase, por ejemplo, MoraLES (2010) p. 30; y Morales (2006) pp. 29 y ss. (especialmente, pp. 33 y ss., en relación con la absorción de la doctrina del riesgo en la noción de incumplimiento). Y en esta misma línea, en Chile, véase, por ejemplo, VIDAL (2009) pp. 232 y ss.; y MejíAs (2011) pp. 89 y ss.

34 Cfr. Stone (2009) p. 632.

35 No está de más decir que esta base histórica del specific performance es compartida por los demás ordenamientos que pertenecen al Common Law, lo cual es debido a la expansión colonial de Gran Bretaña, y a la consecuente implantación/recepción de su Derecho en los distintos territorios que estuvieron bajo su influencia. Para una síntesis de la formación del Common Law (en sentido amplio, como "sistema jurídico"), y particularmente, sobre su extensión a otros territorios, véase, por ejemplo, MORINEAU (2004) capítulos dos y tres; DAVID y JaUfFret-Spinosi (2010) pp. 211 y ss.; o ZWEIGERT y Kötz (1998) pp. 219 y ss.

36 A pesar de que existen notables diferencias entre el Common Law inglés -que comienza a surgir, sobre todo, a partir del siglo XII- y el Derecho anterior (el Derecho "anglosajón" o también denominado el "Derecho de Eduardo", por Eduardo el Confesor, cuya muerte dio origen a la guerra de conquista), estudios recientes sugieren que a los primeros reyes normandos interesó mantener la continuidad del Derecho, y que el proceso de cambio es más bien de "evolución” que de "revolución" [cfr. Thomas (2008) pp. 84 y ss.]. 
minación de este Derecho como "common law", y por extensión, a estos tribunales como "common law courts" 37 .

Aunque las king's courts, y el Derecho "común" que ellas aplicaban, terminaron por desplazar a las diversas cortes locales y al derecho consuetudinario, no se trató de un ataque frontal, sino de un proceso natural ${ }^{38}$. De hecho, en un comienzo su intervención era excepcional: el interesado debía solicitarla al rey, quien, a través de la cancillería, emitía una orden dirigida a la autoridad competente para que resolviera el asunto ${ }^{39}$. Dicha orden se denominaba "writ" ${ }^{40}$. La paulatina estandarización de estos writs dará forma al sistema de acciones conocido como "Forms of actions" o "Writ system", una de las características más importantes del Derecho medieval inglés, y que fue abolido recién en el siglo XIX ${ }^{41}$.

El sistema de acciones - "Forms of actions"-, aunque inicialmente bien recibido, con el tiempo comenzó a mostrar una serie de limitaciones.

Una de las principales limitaciones era su excesiva rigidez. Y es que, si bien el desarrollo inicial de este sistema estuvo marcado por la creación de un writ para cada uno de los derechos o intereses que eran reconocidos por los tribunales, este proceso se detuvo en la segunda mitad del siglo XIII. Desde entonces, las common law courts no admitieron ningún reclamo que no pudiera ser encajado en alguna de las forms of actions o writs ya existentes ${ }^{42}$.

Otra importante limitación era que el common law no reconocía derechos sobre bienes distintos del derecho de propiedad, como por ejemplo, el derecho a usar o disfrutar de un bien determinado. Esta limitación explica el origen de una de las instituciones que, se dice, es una de las más características del Derecho anglosajón, el trust ${ }^{43}$.

37 Sobre el proceso de unificación, luego de la conquista normanda, y en particular, sobre el surgimiento de las king's courts, véase, por ejemplo, Maitland y Montague (1915) pp. 26 y ss.; Van Caenegem (1988) pp. 1-28; y BAKER (2002) pp. 12-34.

38 Según ZWeigerT y KÖTZ (1998) pp. 183-184, ello se debió no solo al prestigio y autoridad de los jueces reales, sino también porque el procedimiento de las cortes reales era más moderno.

39 Cfr. Mourineau (2004) pp. 15 y 16; David y JaufrRet-Spinosi (2010) pp. 215-216.

${ }^{40}$ Originalmente, "writ" designaba cualquier documento oficial escrito, ordenando, prohibiendo o notificando algo [Black's Law Dictionary (2009): "writ"]. De hecho, "writ" significa literalmente "escrito": es una forma arcaica del participio del verbo "to write [PARTRIDGE (1977): "writ"]. Los writs eran utilizados por la monarquía anglosajona, pero como instrumento jurídico, su desarrollo se debe a los normandos $c f r$. VAN CAENEGEM (1988) pp. 30-31. Con el tiempo, adquirió la significación anotada: la de una orden para instruir un proceso judicial cfr. ZWEIGERT y KÖTZ (1998) p. 184.

${ }^{41}$ Sobre este sistema de acciones, véase Maitland (1997) y Plucknett (2001) pp. 353 y ss. Para una exposición más resumida Maitland y Montague (1915) pp. 90 y ss.

42 Cfr. Maitland (1997) pp. 1-5; y Van Caenegem (1988) p. 29 y ss. La mentada limitación a la creación de nuevos writs fue consecuencia del Statute of Westminster II, de 1285 cfr. CARTWRIGHT (2007) pp. 3-8; ELLIOT y QUINN (2009) pp. 115-122. La principal causa de esta limitación es la molestia que generaba en los barones la emisión constante de nuevos writs, lo que implicaba la existencia de un mayor número de leyes, en cuya creación ellos no habían tenido parte c fr. ZWEIGERT y KÖTZ (1998) p. 185. La limitación se encuentra en el Capítulo 24 del mentado estatuto; aunque este preveía un procedimiento regular para la creación de nuevos writs, es claro que los funcionarios de la cancillería no consideraron que el estatuto les diera amplios poderes para crear nuevas forms of actions cfr. Plucknett (2001) pp. 28-29 (con reproducción del texto del mentado Capítulo 24 del Estatuto).

43 Para ilustrar el problema que suponía la falta de reconocimiento por parte del common law de otros derechos sobre los bienes, distintos del de propiedad, suele ponerse el caso de un caballero que parte a las cruzadas, y que quiere proteger sus bienes y asegurar que su esposa e hijos disfruten de ellos en su ausencia. Estos beneficiarios (la mujer y sus hijos) carecían de capacidad para ser titulares de dichos bienes, por tanto, la única solución era que el 
Otra limitación, que interesa particularmente destacar aquí, recaía en los remedios que otorgaban las common law courts. En este sistema, el litigante que obtenía una sentencia favorable tenía únicamente derecho a obtener una indemnización de daños (damages). Sin embargo, la indemnización de daños no siempre era un remedio adecuado. Esta limitación del common law resulta muy relevante en el contexto de este trabajo, pues está estrechamente conectada con la creación del remedio del specific performance, como se verá inmediatamente.

Pues bien, la injusticia que generaban esas y otras limitaciones ${ }^{44}$, llevaron a las personas a presentar sus casos directamente al rey, quien los escuchaba, y resolvía en ejercicio de su poder discrecional, considerando las circunstancias del caso. La atención de estas peticiones fue siendo delegada, paulatinamente, en el Chancellor, dando origen así a la Chancery court. Este tribunal no resolvía de acuerdo con las estrictas reglas del common law, sino conforme a las "rules of equity and good conscience". De ahí la denominación de "equity" a la jurisdicción a la que dio lugar, y por extensión, a los tribunales como equity courts ${ }^{45}$. Con el tiempo, la equity fue adquiriendo importancia, y comenzó a considerarse un sistema separado de Derecho, que complementaba y llenaba los vacíos del common law ${ }^{46}$. Esa doble estructura judicial (common law courts - equity courts) subsistirá hasta el siglo XIX ${ }^{47}$.

caballero dejase sus bienes a una persona de su confianza (de ahí el nombre, trust), para que los administrase en beneficio de sus familiares. El common law no reconocía ningún derecho a los beneficiarios, y la equity vino a llenar ese vacío, reconociendo el valor del trust cfr. por ejemplo, BRAY (2012) p. 3; y STOCKWELl y EDWARDS (2005) p. 6. En todo caso, que el trust sea una institución característica de los ordenamientos del Common Law, como suele entenderse, es una apreciación que, en palabras de Zimmermann (2009) pp. 82-85, "hoy precisa ser revisada", pues, como muestra este autor, existieron muchas instituciones similares al trust en la Edad Media en el continente europeo, y que ellas "dependieron de manera asombrosa del Derecho romano [el que] no había desarrollado, es cierto, un concepto unitario y abstracto de trust, pero había reconocido determinadas formas de fiducia y de cargos de confianza".

${ }^{44}$ Adicionalmente, la jurisdicción de estos tribunales comenzó a percibirse como lenta, muy técnica y cara (cfr. SLAPPER (2011) p. 129). Si el demandante escogía mal el writ que mejor se adaptaba a su pretensión, podía desestimarse la demanda; y la elección entre los diferentes writ no era fácil, debido a su creciente número. Además, cada writ tenía su propias reglas de procedimiento $c f r$. ZWEIGERT y KöTZ (1998) p. 187.

45 Cfr. Elliot y Quinn (2009) pp. 3-8; y CarTwright (2007) pp. 3-8. Es interesante notar que este sistema de acciones, marcado por la necesidad de elegir un writ determinado, recuerda al sistema formulario del Derecho romano; y es igualmente llamativa la similitud entre el surgimiento de la Equity y actividad del pretor. En este sentido, se ha afirmado que la Chancery court "procedía bajo la teoría, tal y como lo hiciera el peregrine praetors con los extranjeros en el derecho romano, de que los principios de derecho natural no formaban parte del sistema legal. No obstante, estos debían ser aplicados por la autoridad civil, por lo menos en ciertas situaciones excepcionales, cuando el sistema legal en cuestión no proporcionara remedio de ninguna especie, o en el mejor de los casos, uno inadecuado" BROWN (1974) pp. 26-27, si bien es bastante claro que no se puede hablar aquí de una recepción del Derecho romano (sobre el punto, véase BucKLAND y MCNAIR (1952) pp. 3-6; y también PÉreZ-RAGONE (2007) pp. 333-356). En todo caso, a pesar de las semejanzas, se ha puesto de relieve que la lógica con que operan ambos sistemas es distinta: la fórmula romana supone un importante desarrollo del derecho sustantivo, mientras que en el sistema del Common Law, como se ha visto, se terminó por entender que un reclamo solo era admisible si se fundaba en un writ determinado $c f r$. WATSON (1990) pp. 253-258.

46 BRAY (2012) p. 4. Aun cuando puede resultar algo difícil de comprender, el que una determinada clase de jurisdicción pueda entrar en el conocimiento de asuntos que corresponden a otra; ello se entiende si se considera el hecho de que "the jurisdiction of equity is a prerogative jurisdiction [...] it is exercised in legal contemplation by the sovereign, who is the fountain from which all justice flows, and from whom, therefore, all courts derive their jurisdiction" LaNDGell (1908) pp. 23-24. Para una presentación sobre el origen y evolución de la Court of Chancery y la Equity, véase BAKER (2002) pp. 97-115.

47 Vuelvo sobre ello más adelante; infra nota 58. 


\subsection{EL ORIGEN DEL REMEDIO DEL SPECIFIC PERFORMANCE}

Suele afirmarse que la orden de specific performance (como también el remedio de la injunction) es una respuesta a la insuficiencia del único remedio que concedía el common law en caso de incumplimiento contractual: el remedio de los damages ${ }^{48}$. Tal afirmación plantea varias preguntas, todas estrechamente conectadas: i) ¿por qué las common law courts únicamente concedían el remedio de damages?; ii) ¿por qué el remedio de damages podía considerarse insuficiente o inadecuado?; y iii) ¿por qué -y en qué medida- la equity podía suplir esa insuficiencia? A continuación, intentaré ofrecer una respuesta a dichas preguntas.

Una posible razón de que las common law courts únicamente concedieran el remedio de damages, es que se trataba de una solución práctica, adaptada a los requerimientos del comercio, cuyo desarrollo está íntimamente ligado al desarrollo del Derecho contractual. En este punto, vale la pena reproducir las palabras de FRY: "[T] he introduction into jurisprudence of any provision for enforcing contracts [was slow] [...] that introduction was due to the increase of commercial activity. The same spirit of commerce which led to the enforcement of contracts, also brought in the notion that money is an equivalent of everything -is an universal measure: and this, coupled with the simplicity of early contracts and the difficulty attendant on the specific performance of complicated ones, probably led to the arrested growth of the remedies for their breach and the confining of such remedies for the most part to the payment of money or the delivery of a chattel” 49 .

Ahora bien, para comprender la razón de que la vía de satisfacción mediante el remedio de los damages podía ser, en más de algún caso, insuficiente o inadecuada, resulta necesario considerar cuál era la forma de coacción de las common law courts. Tras la sentencia, el tribunal dirigía una orden al sheriff, para que este tomara por la fuerza el dinero o los bienes de la parte vencida, a fin de que fueran entregados al litigante vencedor, o bien para ser vendidos con el fin de pagarle con el producto de la venta ${ }^{50}$. La debilidad del remedio es evidente: la satisfacción de la parte vencedora dependía de que el sheriff pudiera efectivamente encontrar el dinero o los bienes, y hacerse con ellos ${ }^{51}$.

Lo que se acaba de indicar sugiere matizar, en cierto sentido, la afirmación de que en las common law courts no fuera posible obtener nada más que una indemnización de daños (damages). Como se puede observar, hay casos en los que el resultado, mirado desde el punto de vista del acreedor, se acerca al del remedio del specific performance. Esto es lo que ocurre en los casos en los que la obligación consistía en pagar una suma de dinero (actio in debt), o en los que consistía en la entrega de determinados bienes (actio in detinue) ${ }^{52}$. La razón para no incluir estos supuestos dentro del grupo de casos en los que se reconoce el specific performance (como remedio frente al incumplimiento contractual), no es solo el limita-

\footnotetext{
48 Cfr. por ejemplo, Cartwright (2007) p. 250; y Zweigert y Kötz (1998) pp. 189-190. En cuanto al momento histórico en que surge el remedio del specific performance, existen testimonios que apuntan a que ya se solicitaba y concedía a mediados del siglo XV, aunque las órdenes de cumplimiento específico se hicieron más comunes a comienzos del siglo XVI. Sobre el punto, véase, WATERMAN (1881) pp. 3-4.

49 FrY (1911) pp. 4-5.

50 Cfr. Landgell (1908) p. 23; Farnsworth (2010) p. 162.

51 Cfr. Landgell (1908) p. 23.

52 Una exposición de estos casos en FrY (1911) pp. 5 y ss.
} 
do alcance de la expresión (en estos casos, aunque se puede decir que el acreedor obtiene lo debido in natura, no se le ha ordenado directamente al deudor cumplir con la prestación), sino que, además, el hecho de que en la base de dichos reclamos está más bien la detentación injustificada de la propiedad, no el incumplimiento de una obligación contractual. En efecto, la mayoría de los writs que podían ser usados en el marco de relaciones comerciales pueden ser considerados como acciones in rem, es decir, acciones que estaban dirigidas a recuperar la propiedad, incluyendo la actio in debt $t^{53}$.

Lo que se acaba de decir permite comprender por qué, en la actualidad, la actio in debt, también llamada action for an agreed sum (que es aquella por la que se pretende la entrega de una suma de dinero debido, por ejemplo, el precio de un contrato o action for the price) no se pueda considerar propiamente un supuesto de specific performance. Si bien en cierto sentido la actio in debt se asemeja al cumplimiento in natura (porque, a diferencia de una acción de daños, en la actio in debt no es necesario probar el daño), la actio in debt, a diferencia del remedio del specific performance, no está sujeta a la discrecionalidad del tribunal, está siempre disponible, y no se ordena pagar bajo apercibimiento de desacato al tribunal ${ }^{54}$.

Tratándose de la orden de entregar bienes muebles -la actio in detinue - la lógica también era propietaria: así, si (A) celebraba un contrato con (B) en virtud del cual este debía entregar un bien a (A), a cambio de una suma de dinero, y (A) pagaba y (B) no entregaba el bien, (A) podía entonces exigir la entrega de dicho bien, pero no alegando el incumplimiento contractual, sino alegando que el contrato se había ejecutado, y por tanto, que el bien era suyo y que (B) lo retenía injustificadamente ${ }^{55}$.

Como se ha dicho antes, la preferencia por el remedio de los damages puede ser explicada, como sugiere FrY, por una lógica de mercado, que ve en el dinero un sustituto adecuado para todo. Pero existe, además, una explicación puramente histórica para esta preferencia. Y es que, sin perjuicio de que el incumplimiento de un acuerdo podía ser la base de una acción in debt o in detinue, no era extraño que los reclamos basados en incumplimiento "contractuales" se encausaran como supuestos de wrongs o trespass (en términos actuales, como pretensiones "extracontractuales"). En estos casos, el demandante no reclamaba el cumplimiento de la prestación, sino una suma (ilíquida) de dinero, por el daño que le había ocasionado la actuación del demandado ${ }^{56}$.

\footnotetext{
53 Cfr. Evers (1977) p. 4.

54 Cfr. Smith y Atiyah (2005) p. 388; Andrews (2011) p. 523. Pese a ello, no es extraño hallar afirmaciones en el sentido de que "el common law did not specifically enforce contractual obligations except those to pay money" Treitel (2003) p. 1019.

55 Cfr. Fry (1911) p. 8; Farnsworth (2010) p. 162.

56 Cfr. IBbetson (1997) pp. 1-3. El estudio que el autor realiza sobre estos juicios durante el siglo XIII, le permite concluir que no es extraño ver que se reclama, en sede "extracontractual" (tort), el hecho de que el incumplimiento del contrato por el deudor ha generado deshonor o vergüenza al acreedor (víctima), vinculando la pretensión de resarcimiento a la antigua acción de trespass, acción que tenía por objeto la compensación por las pérdidas sufridas (aun cuando los daños se calcularan por referencia a las expectativas pérdidas). Sobre la acción de trespass, véase BAKER (2002) p. 60 y ss. El Derecho contractual del Common Law se desarrolla posteriormente, precisamente a partir del writ of assumpsit, que es una forma de trespass (para una síntesis sobre ello, en
} 
Por último, hay que considerar que, ante la insuficiencia o inadecuación del remedio de los damages del common law, la equity ofrecía una alternativa, porque la vía de coacción de estos tribunales era diferente a la de las common law courts. En efecto, la ejecución de las sentencias de las courts of equity tomaron, desde un comienzo, la forma de una orden dirigida directamente al demandado, ordenándole hacer o no hacer algo, bajo apercibimiento de sancionarle con multas, con la toma de sus bienes o incluso con prisión, debido en una parte importante, a la influencia del Derecho canónico ${ }^{57}$.

\section{EL SIGNIFICADO ACTUAL DEL CARÁCTER EQUITABLE DEL REMEDIO DEL SPECIFIC PERFORMANCE. LAS BARRERAS AL CUMPLIMIENTO ESPECÍFICO}

Lo primero que hay que tener presente, es que la mentada doble estructura judicial (common law courts - equity courts) ha sido casi completamente abandonada en los países del Common Law ${ }^{58}$.

No obstante ello, subsiste la distinción entre "reglas del common law" y "reglas de equity" 59 . Y, por esta razón, el adjetivo "equitable" con el que suele caracterizarse al remedio

conexión con el remedio de cumplimiento específico, véase ZIMMERMANN (1990) pp. 776 y ss.; y BAKER (2002) pp. 329 y ss.].

57 Esta vía de coacción -una orden dirigida directamente al deudor- tendría su origen en la jurisdicción canónica, cuya influencia sería explicada debido a que los primeros cancilleres eran clérigos [cfr. FARNSWORTH (2010) p. 163]. Según Langdell (1908) pp. 23-25, los primeros cancilleres se enfrentaron a un problema similar al de las cortes eclesiásticas, y la solución adoptada es similar: la jurisdicción canónica únicamente tiene poder "espiritual", y por tanto, tiene que obtener el cumplimiento de sus sentencias por parte del propio condenado, bajo la amenaza de sanciones religiosas (como la excomunión); de forma similar, la equity tenía únicamente un poder "físico", y no tenía poder legal y, en consecuencia, aunque podía, por ejemplo, tomar bienes del deudor por la fuerza, ello no afectaba al título de propiedad, siendo, por tanto, una solución insatisfactoria. Por ello, lo que había que obtener era que el propio deudor cumpliera con la prestación prometida. Para una exposición sobre el origen de la jurisdicción eclesiástica y en particular, en relación con el origen del remedio de specific performance, véase FrY (1905) pp. 8-15, quien también sugiere la posible influencia de la jurisdicción canónica en la adopción del remedio del specific performance en la Chancery Court.

${ }^{58}$ En el caso del Derecho inglés, las common law courts y las equity courts se fusionaron en una sola estructura judicial en virtud de los Judicature Acts 1873-1875 cfr. CARTWRIGHT (2007) pp. 6-7. Por extensión, también se produjo en los demás países de la Commonwealth. En Australia, por ejemplo, los Judicature Acts 1873-1875 fueron promulgados en todos los estados, salvo en New South Wales, que conservó una court of equity separada hasta 1972 [sobre el punto, véase BRYAN y VANN (2012) p. 4; quienes ofrecen, además, un resumen de la evolución de la equity, los principales motivos que justificaron la reforma y los principales cambios que implicó, pp. 7-10]. En el caso de EE.UU., si bien la separación subsiste en algunos estados, la mayoría de ellos ha fusionado estos tribunales en un sistema judicial único; fusión que también existe a nivel federal [cfr. KLAss (2010) p. 25; y Brown (1974), especialmente, pp. 28 y ss.]. Para una presentación general sobre el proceso de fusión en EE.UU., véase McCormick (1928) pp. 283-298.

59 Existe cierto debate en torno al alcance de los Judicature Acts 1873-75. La posición ampliamente mayoritaria -tanto en Inglaterra como en los demás países de la Commonwealth- es que su finalidad únicamente alcanza a la forma de organización de la administración de justicia, y no afecta a las reglas sustantivas, de modo que la distinción subsiste $c f r$. CARTWRIGHT (2007) p. 7. Así, el único efecto es que, desde su entrada en vigor, cualquier tribunal puede y debe decidir los casos sobre la base del "common law" y de la "equity" cfr. SLapper (2011) p. 131. La forma en que se relacionan estas dos clases de reglas suele ser expresada recurriendo a la metáfora de ASHBURNER "[ $t]$ he two streams of jurisdiction, though they run in the same channel run side by side and do not 
del specific performance, no es un mero residuo lingüístico: el origen histórico de este remedio - del que se acaba de dar cuenta- permite explicar las "barreras" o restricciones que existen a la hora de concederlo en la actualidad ${ }^{60}$.

Esas "barreras" son, básicamente, dos: la primera es que el specific performance solo se concede en caso de que el remedio de los damages se considere inadecuado; la segunda, reside en el carácter discrecional del remedio del specific performance. De ellas doy cuenta a continuación.

\subsection{LA ADECUACIÓN DEL REMEDIO DE LOS DAMAGES PARA SATISFACER AL ACREEDOR}

\section{$A$. El adecuacy test y carácter "único" del objeto como principal criterio}

La principal limitación del remedio del specific performance es, como ya se ha dicho, que este solo se ordenará en la medida que se considere que el remedio de los damages sería “inadecuado". En efecto, en la medida que el acreedor puede procurarse un sustituto del objeto debido, acudiendo al mercado, se entiende que su interés en el cumplimiento está suficientemente protegido por esta vía: basta con que la parte incumplidora indemnice al acreedor, asegurando el beneficio que le otorgaba el contrato original ${ }^{61}$.

El remedio del specific performance está justificado, por tanto, si no es posible procurarse un sustituto o, dicho de otro modo, en la medida en que el objeto del contrato sea "único" ${ }^{2}$. El caso típico en el que se considera que el objeto es "único" son los contratos re-

mingle their waters. The distinction between legal and equitable claims - between legal and equitable defences -and between legal and equitable remedies- has not been broken down in any respect by recent legislation" [(1902) p. 18] Utilizando la misma metáfora, Lord Diplock, en United Scientific Holdings Ltd v. Burnley Borough Council [(1978) AC 904] señaló -representando la visión minoritaria- que "waters of the confluent streams of law and equity have surely mingled now". Sobre este debate, véase, por ejemplo, BuRns (1993) y también MAXTon (1993), quien sugiere, tras su análisis, que efectivamente existe cierta fusión de las reglas.

En relación con el Derecho estadounidense, la conclusión es la misma. La unificación del sistema judicial no afecta a la distinción sustantiva; de hecho, se afirma que continúa modelando el Derecho contractual, y, en particular, explica la preferencia por el remedio de los damages en casos de incumplimiento contractual [c $f r$. VON Mehren y Murray (2007) pp. 2-3; en el mismo sentido, Klass (2010) p. 25)]. La distinción también interesa para otros fines: por ejemplo, para determinar si existe o no, en conformidad con la $7^{\mathrm{a}}$ enmienda de la Constitución de EE.UU., derecho a un jurado: si la acción puede ser considerada como una acción de "equity", no lo hay [cfr. BURHAM (2006) pp. 239-240].

${ }^{60}$ Así, Smith y Atiyah (2005) p. 388; Cartwright (2007) p. 7; en el mismo sentido, en relación con el Derecho neozelandés, CunCANNON (2004) p. 660. Para profundizar sobre la justificación de la conservación de estas "etiquetas" históricas en el Derecho inglés actual, véase Burrows (2002) pp. 1-16.

${ }_{61} C f$ r. CARTWRight (2007) p. 256. En consecuencia, en la medida que no exista variación en el precio del mercado, la víctima del incumplimiento no obtendrá nada, y el deudor no enfrentará ninguna sanción legal [cfr. Smith y Aтiуah (2005) pp. 371-372].

62 Cfr. por ejemplo, Andrews (2011) pp. 530-531, quien afirma que, en esos casos, el cumplimiento específico es el "remedio primario". El criterio ha sido recogido expresamente en la s. 52 del Sale of Goods Act 1979 (Specific performance): "In any action for breach of contract to deliver specific or ascertained goods the court may, if it thinks fit, on the plaintiff's application, by its judgment or decree direct that the contract shall be performed specifically, without giving the defendant the option of retaining the goods on payment of damages".

En el Derecho estadounidense, el \$2-716 UCC (Buyer’s Right to Specific Performance or Replevin) recoge el mismo principio, en términos parecidos: "(1) Specific performance may be decreed if the goods are unique or in other proper circumstances. In a contract other than a consumer contract, specific performance may be decreed if the parties 
lativos a bienes raíces (debido a que son, por su propia naturaleza, bienes “únicos”) ${ }^{63}$. Pero existen otros ejemplos, como la compra de antigüedades o reliquias ${ }^{64}$, la compra de acciones de una sociedad ${ }^{65}$, o las adquisiciones de patentes y derechos de autor ${ }^{66}$. En todos estos casos, el remedio de los damages es inadecuado, bajo la lógica de que el interés del acreedor no es medible en términos de dinero $^{67}$, o mejor dicho, porque se considera imposible, en estos casos, determinar cuál es el monto apropiado ${ }^{68}$.

have agreed to that remedy. However, even if the parties agree to specific performance, specific performance may not be decreed if the breaching party's sole remaining contractual obligation is the payment of money". Por su parte, el $\mathbb{\$}$ 360 Rest (2d) CONTRACTS (Factors Affecting Adequacy of Damages), recoge tres criterios importantes a la hora de determinar la adecuación o no del remedio de los damages: $i$ ) la dificultad de probar la existencia de daños con razonable certidumbre, ii) la dificultad de procurar un sustituto adecuado por la vía del dinero y iii) la probabilidad con que se pueda obtener la suma de dinero a la que se condene ["In determining whether the remedy in damages would be adequate, the following circumstances are significant: /(a) the difficulty of proving damages with reasonable certainty, / (b) the difficulty of procuring a suitable substitute performance by means of money awarded as damages, and /(c) the likelihood that an award of damages could not be collected"]. Sobre la justificación del criterio del carácter "único" del objeto del contrato en la doctrina estadounidense, véase KronMAN (1978) pp. 355365. Sobre el principio de la "especificidad" o del carácter "único" del objeto de la prestación, en relación con el Derecho canadiense, véase STACK (2000) p. 62.

${ }_{63}$ Cfr. en relación con el Derecho inglés, Samuel (2001) p. 162; en relación con el Derecho estadounidense, FARnsworth (2010) p. 176; y en relación con el Derecho australiano, CARTer et al. (2007) p. 939. Sin embargo, es posible pensar que otra razón para favorecer el cumplimiento específico en estos contratos es la importancia social y económica que se atribuía a estos bienes en la época en que se desarrollan las doctrinas de equity en este sentido, WilLinstone (1920) p. 2515.

${ }^{64}$ El caso paradigmático es Pusey v. Pusey, de 1684 [23 Eng. Rep. 465], en el que se ordenó el cumplimiento específico consistente en la transferencia de un antiguo cuerno (que en la actualidad se encuentra en el Victoria and Albert Museum, en Londres), que había sido dado a la familia Pusey por el Rey danés Canuto el grande, en el siglo XI. Otro ejemplo es Falcke v. Gray de 1859 [62 Eng. Rep. 250], en el que la solicitud de cumplimiento específico tiene como fundamento el incumplimiento de un contrato de compraventa de dos jarrones chinos. La Corte consideró, en este caso, que debido a su "inusual belleza, rareza y distinción", la indemnización de daños no sería una compensación adecuada [ambos casos citados por FrY (1911) pp. 37-38, y por KronMANN (1978) p. 356].

65 Cfr. Andrews (2011) p. 531.

${ }^{66}$ Cfr. Farnsworth (2010) p. 176.

${ }^{67}$ El punto puede ser bien ilustrado con las palabras de Lord Nicholls en Attorney General v. Blake and Another (2000) UKHL 45]: "It is [...] well established that an award of damages, assessed by reference to financial loss, is not always "adequate" as a remedy for a breach of contract. The law recognises that a party to a contract may have an interest in performance which is not readily measurable in terms of money [...] The classic example of this type of case [...] is a contract for the sale of land. The buyer of a house may be attracted by features which have little or no impact on the value of the house. An award of damages, based on strictly financial criteria, would fail to recompense a disappointed buyer for this head of loss. The primary response of the law to this type of case is to ensure, if possible, that the contract is performed in accordance with its terms".

${ }^{68}$ En este sentido, Sмітн у АтіYан (2005) p. 380, quienes explican que es difícil de justificar que, en estos casos, la inadecuación del remedio de los damages se deba a que ninguna suma de dinero será suficiente compensación. En la realidad, siempre existe una suma de dinero que compensará completamente al acreedor. En efecto, dicen estos autores, quien compra una casa, estará más que feliz de recibir diez veces el precio pactado. Por ello, sugieren que es más plausible sostener que, en estos casos, el remedio de damages es inadecuado porque es casi imposible determinar cuál es la suma adecuada. 


\section{B. La tendencia a la flexibilización}

Este "adecuacy test" es, como se puede apreciar, una limitación importante a la hora de conceder el specific performance. No obstante, hay que tener presente que parece existir cierta tendencia a la flexibilización de este criterio.

Una manifestación de esa tendencia a la flexibilización es la admisión del remedio del specific performance tratándose de obligaciones de dar cosas fungibles, obligaciones en las que, por definición, quedaría excluido ese remedio (no son bienes únicos, siempre existe un sustituto). Se ha entendido que el specific performance podría estar justificado en la medida que dichos bienes escaseen en un momento determinado. En la doctrina inglesa suelen citarse dos casos, relativamente recientes, que reconocen que el mercado -a pesar de que normalmente ofrece una vía alternativa de suministro de los bienes- puede fallar en un momento determinado, justificando ello el cumplimiento específico. Uno es Sky Petroleum Ltd v. VIP Petroleum Ltd [(1974) 1 WLR 576], en el que se ordenó la entrega de unas cantidades de petróleo; y el otro es Perry \& Co v. British Railways Board [(1980) 2 All ER 579], en el que se ordenó la entrega de unas cantidades de acero. Como se puede observar por las fechas de ambos juicios, la posibilidad de obtener un sustituto en el mercado era casi imposible: el primer juicio se enmarca en la llamada "crisis del petróleo" de 1973, y el segundo, en la llamada "huelga del acero", que afectó al Reino Unido en 1980. Así, pues, el carácter "único" del objeto del contrato puede también ser apreciado desde una perspectiva "de mercado" ${ }^{69}$, flexibilizando el criterio que permite entender que los damages son inadecuados. Sin embargo, hasta qué punto los tribunales lo entienden así es algo difícil de determinar. Por ejemplo, en un caso de 1804 se ordenó el specific performance de la obligación consistente en suministrar una máquina, porque no era posible para el acreedor procurarse un sustituto fácilmente en el mercado ${ }^{70}$; sin embargo, en un caso similar, en 1975, se negó el specific performance, aun cuando en el caso concreto era evidente que al acreedor tomaría más de un año obtener dicha máquina de otro suministrador ${ }^{71}$.

Puede considerarse como otra manifestación de la mentada tendencia a la flexibilización en la aplicación del criterio de la especificidad del objeto del contrato, el que hoy se plantee en la doctrina la pregunta sobre si el carácter "único" o "singular" debe depender exclusivamente de elementos objetivos, o si también debería considerarse el valor que le atribuya el acreedor ${ }^{72}$. Ahora bien, el considerar la intención del acreedor o de las partes en la determinación del carácter "sustituible" del objeto, no necesariamente implica favorecer el specific performance; al contrario, ello puede tener como efecto el restringir la concesión de

69 Cfr. en relación con el Derecho inglés: ANDREws (2011) pp. 531-532 (recogiendo la opinión de otros autores); CARTwright (2007) p. 254; Treitel (2003) pp. 1022-1023. En el mismo sentido, en relación con el Derecho estadounidense, FARNSWORTH (2010) p. 178. En el Derecho canadiense también se considera "único" un bien, aun cuando objetivamente sea fungible, en la medida que haya escasez cfr. STACK (2000) p. 62.

70 Así se resolvió en Nutbrown v. Thornton [(1804) 10 Ves. 159; citado por Treitel (2003) p. 1023.

71 Así, en Societé des Industries Metallurgiques SA v. Bronx Engineering Co. Ltd [(1975) 1 Lloyd's Rep 465 CA]; un resumen de este caso puede verse en Oughton y Davis (2000) pp. 528-530.

72 Cfr. AndRews (2011) p. 531. LANDGELl (1908) p. 49, plantea dos objeciones para no considerar estos factores subjetivos (la valoración del deudor): la primera, es que admitir la prueba de ello en el juicio envuelve gastos, tanto de las partes como de la administración judicial; la segunda, es que supone al tribunal investigar un punto sobre el cual difícilmente podría llegar a establecer la verdad, y por tanto, sobre el que no debería entrar. 
este remedio. Esto es lo que puede ocurrir, por ejemplo, en el caso de la compra de un inmueble. Como se ha dicho, tradicionalmente se entiende que en este caso procede ordenar el specific performance; pero, si se toman en cuenta los motivos de la compra del inmueble, por ejemplo, el que se esté comprando para revender, entonces la procedencia del specific performance podría resultar más difícil de justificar ${ }^{73}$.

Por último, se advierte cierta tendencia, por parte de los tribunales, a conceder el remedio del specific performance en forma más amplia. En este sentido, un sector de la doctrina inglesa ha sugerido que los tribunales ya no se preguntarían tanto si el remedio de los damages es o no adecuado, sino cuál remedio es el "más apropiado" para el caso concreto. Quienes así lo afirman encuentran su fundamento en el caso Beswick v. Beswick [(1968) UKHL 2]. Los hechos de Beswick v. Beswick son, en términos muy simples, los siguientes. A transfirió su negocio a $B$ (su sobrino) a cambio de que B le pagara $6.10 £$ a la semana mientras viviera, y en caso de morir A, pagara, anualmente, la suma de $5 £$ a la semana a su viuda C. A muere al poco tiempo, B pagó una vez a C $5 £$ y se negó a pagar más. C demanda las sumas adeudas hasta el momento (175£) y solicita una orden de cumplimiento específico para que se continuara pagando la anualidad, lo que hace en nombre propio y como administradora del patrimonio de su marido. La Court of Appeal le da la razón, en ambas condiciones. La House of Lords consideró que C, si bien no tenía derecho a demandar en nombre propio (como tercero beneficiario), sí podía demandar en su condición de administradora del patrimonio de A. El hecho de que el incumplimiento de B no dañara el patrimonio de A significaba que únicamente podía obtener "nominal damages", y como ello era injusto, se ordenó el cumplimiento específico. Las palabras de Lord Hodson expresan con claridad este "giro" jurisprudencial: "[t] he only question is, What is the appropriate reme-

\footnotetext{
73 Así, en relación con el Derecho estadounidense, Farnsworth (2010) p. 176. Aunque la naturaleza "única" del inmueble no determina el derecho al cumplimiento específico (este remedio siempre es discrecional), justifica una presunción de que el remedio de daños no es adecuado. Así lo recoge expresamente el Código civil de California, que entiende que esta presunción tiene el carácter de presunción de derecho (iuris et de iure) únicamente si se trata de la compra de una vivienda destinada al uso de una familia, cuando lo que se pretende es precisamente dicho uso. En los demás casos, la presunción admite prueba en contrario [s. 3387 CC de California: "It is to be presumed that the breach of an agreement to transfer real property cannot be adequately relieved by pecuniary compensation. In the case of a single-family dwelling which the party seeking performance intends to occupy, this presumption is conclusive. In all other cases, this presumption is a presumption affecting the burden of proof']. En Canadá, los tribunales ya no conceden automáticamente el cumplimiento específico en el caso del incumplimiento de un contrato de venta de un inmueble, sino únicamente cuando pueda considerarse que es un inmueble "único". El cambio de giro se debe a la sentencia de la Corte Suprema de Canadá en Semelhago v. Paramadevan [(1996) 2 S.C.R. 415] en la que se sostuvo, a pesar de tratarse de la compra de un inmueble, que "the subject property was not unique. It was a building lot under construction which would be interchangeable in all likelihood with any number of others". STACK (2000), sobre esta sentencia, explica, además, que ella es relevante en otros dos aspectos relativos al cumplimiento específico. Por un lado, de la sentencia se desprende que "a successful plaintiff may elect at trial to take damages in lieu of specific performance. This compensation should equal any losses between breach and the trial; therefore, the plaintiff has no duty to mitigate damages", y además, que los "damages in lieu of specific performance are to be a full replacement for performance" [las afirmaciones reproducidas están en la p. 60; la justificación de estas afirmaciones, en las pp. 70 y ss.].
} 
$d y$ ? It would be strange if the only remedy were nominal damages recoverable at common law or a series of actions at law to enforce the performance of a continuing obligation"74.

No obstante, otras autorizadas voces en la doctrina inglesa advierten que no es posible hablar de un cambio significativo en relación con este criterio, descartando que la sentencia de Beswick v. Beswick pueda ser tomada como base para sostener un cambio jurisprudencial, y que el criterio de la adecuación de daños ha sido reafirmado recientemente, citando al efecto el caso Co-operative Insurance Society Ltd v. Argyll Stores [(1997) UKHL $17]^{75}$, a la que me refiero un poco más adelante.

También en la doctrina estadounidense se ha sugerido que los tribunales ya no se preguntarían tanto si el remedio de los damages es o no adecuado, sino cuál remedio es "más apropiado"76. Una evolución similar parece existir en el Derecho australiano ${ }^{77}$.

\subsection{EL EJERCICIO DE LA DISCRECIONALIDAD POR PARTE DEL TRIBUNAL}

Además de la barrera que supone la consideración de que el remedio de los damages es, por regla general, más adecuado que el del specific performance, la otra gran limitación a la hora de conceder este remedio reside en su carácter discrecional. Esto significa que, incluso en los casos en los que el remedio de los damages pueda ser inadecuado, es el tribunal quien decide si procede o no conceder el remedio del specific performance. El specific performance no es un derecho del acreedor.

En este punto, conviene destacar que la incidencia del Derecho privado europeo, sobre esta aproximación al remedio del specific performance del Derecho contractual inglés, es muy limitada. En efecto, la Directiva 1999/44/CE establece, para las ventas de consumo, la sustitución y la reparación del bien como remedios primarios [art. 3.2 $]^{78}$, lo que puede

\footnotetext{
74 Para una exposición de esta sentencia, véase, Mulcahy (2008) p. 207; Ougthon y Davis (2000) p. 523. También Smith y Атіуан (2005) p. 381), quienes sostienen con claridad que la sentencia de Beswick v. Beswick es efectivamente una innovación, puesto que, en el caso, el remedio de damages no era inadecuado, simplemente se estimó que el cumplimiento específico era "más adecuado". Esta visión más liberal representa, en opinión de Treitel (2003) pp. 1025-1026), “una aproximación más satisfactoria”.

75 Cfr. Andrews (2011) p. 533; Cartwright (2007) p. 252; Samuel (2001) pp. 162-163; Stone (2009) p. 635.

76 Así, KLASs (2010) p. 197: "While there is a theoretical preferencce for monetary relief, specific performance is available in a significant number of circumstances [...] [c]ommentators have also observed an increasing judicial willingness to award specific performance, and some have argued that it is in fact more readily available than most doctrinal formulations suggest". FARNSWORTH (2010) p. 938, también afirma la existencia de una "tendencia a la liberalización" del remedio de specific performance, en el sentido apuntado.

77 Cfr. CARTer et al. (2007) p. 938. Resulta interesante apuntar que en el primer semestre del año 2012, el Gobierno australiano inició un proceso de consultas en orden a revisar y modernizar el Derecho contractual. Una de dichas consultas específicas tiene que ver precisamente con el rol del cumplimiento específico como remedio en caso de incumplimiento [sobre ello véase el documento "Improving Australia's Law and Justice Framework. A discussion paper to explore the scope for reforming Australian contract law", y las consultas específicas (incluida la relativa a los remedios frente al incumplimiento), disponible todo ello en la página web del gobierno australiano: [http://www.ag.gov.au/Consultations/Pages/ReviewofAustraliancontractlaw.aspx].

78 El art. 3.2 Directiva 1999/44/CE (Derechos del consumidor) dispone: "En caso de falta de conformidad, el consumidor podrá exigir que los bienes sean puestos en conformidad mediante la reparación o la sustitución del bien sin cargo alguno, de conformidad con el apartado 3, o una reducción adecuada del precio o la resolución del contrato respecto de dicho bien, de conformidad con los apartados 5 y 6 . El mentado apartado 3 dispone: "En primer lugar, el consumidor podrá exigir al vendedor que repare el bien o que lo sustituya, en ambos casos
} 
considerarse como equivalente a exigir del vendedor el specific performance. Pero, como señala TwIGg-FLESNER, la evidente colisión entre la aproximación del Derecho inglés y el enfoque de la Directiva 1999/44/CE se ha resuelto, a la hora de implementar esta última, conservando la aproximación tradicional del Derecho inglés, y por tanto, sin dar pleno efecto a las exigencias de la Directiva: se reconoce al tribunal un poder discrecional para ordenar la sustitución o reparación del bien, pero igualmente se permite al tribunal ordenar otro remedio, si es que lo considera más apropiado ${ }^{79}$.

Ahora bien, que el specific performance sea un remedio "discrecional" no significa que la decisión sobre su otorgamiento dependa "del capricho del juez" ${ }^{80}$. Desde la segunda mitad del siglo XVII, los criterios de decisión adoptados por las equity courts fueron unifor-

sin cargo alguno, salvo que ello resulte imposible o desproporcionado./ Se considerará desproporcionada toda forma de saneamiento que imponga al vendedor costes que, en comparación con la otra forma de saneamiento, no sean razonables, teniendo en cuenta:/ -el valor que tendría el bien si no hubiera falta de conformidad,/ -la relevancia de la falta de conformidad, y/ -si la forma de saneamiento alternativa pudiese realizarse sin inconvenientes mayores para el consumidor./ Toda reparación o sustitución deberá llevarse a cabo en un plazo razonable y sin mayores inconvenientes para el consumidor, habida cuenta de la naturaleza de los bienes y de la finalidad que tuvieran los bienes para el consumidor". El carácter preferente de la reparación y la sustitución queda claro en atención a lo dispuesto por el mentado apartado 5 del art. 3 Directiva 1999/44/CE: "El consumidor tendrá derecho a una reducción adecuada del precio o a la resolución del contrato:/ si no puede exigir ni la reparación ni la sustitución, o/ si el vendedor no hubiera llevado a cabo el saneamiento en un plazo razonable, o/ si el vendedor no hubiera llevado a cabo el saneamiento sin mayores inconvenientes para el consumidor".

79 "There is [...] a very obvious clash between the reluctance of English law to order specific performance and the Directive's focus on repair and replacement as the main remedies in consumer sales contracts. The implementation of the Directive might therefore have necessitated a fundamental change to English practice, albeit confined to the context of consumer sales. Whilst making specific performance more widely available may be a positive step, doing so merely for one particular type of contract seems unattractive. The solution adopted in the implementing legislation has been to give the courts the power to order specific performance of either repair or replacement in the context of consumer sales, but the court is also able to order an alternative remedy if this is more appropriate. This seems to reflect the existing approach of English law, and does not give full effect to the requirements of the Directive" TwIGG-FLesner (2008) p. 136; cursivas añadidas. En el mismo sentido, MAK (2008) p. 128. La implementación de estos remedios de reparación y reemplazo, de la Directiva 1999/44/CE, se ha hecho introduciendo un nuevo apartado en la Sales of goods Act 1979, apartado 5A, titulado "Additional rights of buyer in consumer cases" (s. 48A a s. 48F). La s. 48B (Repair or replacement of the goods) dispone: "(1) If section $48 A$ above applies, the buyer may require the seller / (a) to repair the goods, orl (b) to replace the goods. I (2) If the buyer requires the seller to repair or replace the goods, the seller mustl (a) repair or, as the case may be, replace the goods within a reasonable time but without causing significant inconvenience to the buyer; ( $b$ ) bear any necessary costs incurred in doing so (including in particular the cost of any labour, materials or postage)./ (3) The buyer must not require the seller to repair or, as the case may be, replace the goods if that remedy is/ (a)impossible, orl (b) disproportionate in comparison to the other of those remedies, orl (c) disproportionate in comparison to an appropriate reduction in the purchase price under paragraph (a), or rescission under paragraph (b), of section 48C (1) below./ (4) One remedy is disproportionate in comparison to the other if the one imposes costs on the seller which, in comparison to those imposed on him by the other, are unreasonable, taking into account/ (a) the value which the goods would have if they conformed to the contract of sale, / (b) the significance of the lack of conformity, and/ (c) whether the other remedy could be effected without significant inconvenience to the buyer./ (5) Any question as to what is a reasonable time or significant inconvenience is to be determined by reference tol (a) the nature of the goods, andl (b)the purpose for which the goods were acquired".

80 Cfr. Furmston (2007) p. 798. 
mándose ${ }^{81}$, y hoy constituyen una serie de reglas y principios bien establecidos ${ }^{82}$. Paso a dar cuenta de ellos.

\section{A. La necesidad de supervisión por parte del tribunal}

Si la orden del specific perfomance conlleva la necesidad de una supervisión por parte del tribunal, entonces es altamente probable que se niegue este remedio ${ }^{83}$. Lógicamente, se trata de una limitación significativa tratándose de obligaciones de hacer, y particularmente importante tratándose de obligaciones de ejecución continuada en el tiempo, ya que en estos casos la orden del specific performance depende, en el fondo, de que pueda establecerse con claridad qué es lo que debe realizar el deudor ${ }^{84}$.

Este criterio se encuentra en la base de la decisión del caso Co-operative Insurance Society Ltd v. Argyll Stores [(1997) UKHL 17], uno de los casos más citados por la doctrina actual. Los hechos de este caso son, resumidamente, los siguientes: Argyll decidió cerrar el supermercado que tenía instalado en un local de un centro comercial, debido a que estaba perdiendo dinero. Al hacerlo, incumplió el contrato de arrendamiento celebrado con Cooperative Insurance Society Ltd (el arrendador), que contenía una cláusula expresa por la que Argyll se obligaba a mantener el local abierto durante las horas usuales del comercio. El juez de primera instancia -rechazando la pretensión del arrendador de que se ordenara el specific performance- determinó la procedencia de una indemnización de daños a favor del arrendador. La Court of Appeal, revocando la sentencia, decretó el cumplimiento específico: esto es, ordenó a Argyll continuar operando el negocio hasta la llegada del plazo pactado, o bien hasta que Co-operative Insurance Society Ltd celebrara otro contrato de arrendamiento del mismo local.

En este caso, la House of Lords comparte la justificación del juez de primera instancia para negar el cumplimiento específico tratándose de la obligación de hacer, particularmente, la de mantener en funcionamiento un negocio: "He [se refiere al juez de primera instancia] gave reasons why he thought that specific performance would be inappropriate. Two such reasons were by way of justification for the general practice. An order to carry on a business, as opposed to an order to perform a "single and well defined act" was difficult to enforce by the

81 Desde sus orígenes y hasta el siglo XVII, la concesión de este remedio dependía exclusivamente de la idea de "justicia” que tuviera el Chancellor de turno. Ello puede ilustrarse con la afirmación de un importante jurista inglés del siglo XVII, quien, en el punto de más alta tensión entre la equity y el common law, y a modo de crítica, declaró que "Equity varies with the length of the Chancellor's foot". Sin embargo, a mediados del siglo XVII los casos resueltos por la Cancillería comenzaron a ser publicados, y desde entonces, citados y seguidos en casos posteriores. Se comenzó a entender, pues, que el ejercicio de la discrecionalidad por parte del Chancellor, más que estar limitado por su "consciencia" estaba limitado por el precedente [cfr. PeTtit (2012) p. 5].

En esta línea de objetivación, resulta muy interesante considerar la influencia que pudo haber tenido el cambio de paradigma social y jurídico que se produce entre los siglos XVIII y XIX: la idea de presentar el Derecho como una "ciencia", llevó naturalmente a la objetivación y racionalización de los criterios de decisión de los tribunales. Sobre ello, véase el trabajo de Berryman (1985) pp. 295-323.

82 Como lo ha sostenido Lord Hoffmann: "The principles upon which English judges exercise the discretion to grant specific performance are reasonably well settled and depend upon a number of considerations, mostly of a practical nature, which are of very general application" [Co-operative Insurance Society Ltd v. Argyll Stores (1997) UKHL 17].

83 Cfr. Smith y АтiYah (2005) pp. 382-383; Andrews (2011) p. 532.

84 Cfr. Oughton y Davis (2008) pp. 530-531; y en el mismo sentido, Stone (2009) pp. 634-635. 
sanction of committal. And where a business was being run at a loss, specific relief would be "too far reaching and beyond the scope of control which the court should seek to impose." The other two related to the particular case. A resumption of business would be expensive (refitting the shop was estimated to cost over $£ 1$ million) and although Argyll had knowingly acted in breach of covenant, it had done so "in the light of the settled practice of the court to award damages." Finally, while the assessment of damages might be difficult, it was the kind of exercise which the courts had done in the past'85.

La necesidad de supervisión, o el problema de determinar con claridad qué es lo que el deudor debe hacer, son también justificaciones para negar el specific performance tratándose de otras obligaciones de hacer, como por ejemplo, las surgidas de contratos de construcción (si bien en estas, además, suele considerarse que el remedio de los damages es un remedio adecuado $)^{86}$.

Por último, cabe apuntar que se ha sugerido la existencia de una cierta flexibilización en relación con este criterio, en el sentido de que la necesidad de supervisión por parte del tribunal no sería un factor determinante para negar el specific perfomance, sino que sería meramente un factor a tomar en consideración ${ }^{87}$.

\section{B. La prestación de servicios personales}

Los tribunales son igualmente reacios a ordenar el specific performance de contratos relativos a "servicios personales" ${ }^{8}$. La limitación en la concesión de este remedio alcanza a ambas partes, aunque la justificación es distinta.

\footnotetext{
85 Esta sentencia es interesante en varios aspectos: i) expone la "barrera" al cumplimiento específico basada en la necesidad de supervisión tratándose de contratos en los que el deudor debe una actividad; ii) reafirma el criterio de la adecuación de los daños, aun cuando su determinación pueda ser difícil en algún momento; y iii) propone, además, una distinción entre contratos en los que la obligación "de hacer" consiste en realizar una actividad (prolongada en el tiempo), y entre aquellos en los que consiste en obtener un resultado (caso en el cual, la objeción de la dificultad en la supervisión no es procedente). Para una exposición de este caso, y de su relevancia en relación con la concesión del remedio del specific performance véase, por ejemplo, ANDREWs (2011) pp. 534-537.

86 Treitel (2003) p. 1035. Sobre la relación de esta "barrera" y los contratos de construcción, véase BERRYMAN (1985) pp. 316-319, quien pone de relieve tanto la falta de uniformidad en la decisión de conceder el remedio del specific performance, como el hecho de que la negativa se suele justificar sobre diferentes bases.

87 En este sentido, MulCahy (2008) p. 207, señala: “[h] owever, in some such cases specific performance has been ordered and it has been suggested that the difficulty of supervision is sometimes exaggerated. There are various devices that the court can adopt to overcome such difficulty, such as appointing a receiver and manager. Moreover, there are several judicial statements in recent cases to suggest that "constant supervision» is no longer a bar to specific performance but merely a factor to be taken into account".

${ }^{88}$ Los tratados y manuales de Derecho contractual consultados -a los que se ha hecho referencia a lo largo de este trabajo- no especifican qué debe entenderse por contratos que consistan en realizar "personal services". Entre los rasgos distintivos de un contrato de "servicios personales" se puede mencionar la necesidad de que las partes mantengan una relación personal; que la finalidad del contrato no consiste únicamente en obtener un resultado particular, sino que importa también la forma en que las partes actúan durante la ejecución del contrato; la existencia de información confidencial; y la presencia de deberes de fidelidad [cfr. MulHeron (1999) p. 32].

Los contratos de trabajo (relación laboral) quedan lógicamente dentro de esta categoría de los personal services, pero están fuera del marco de nuestro estudio. En todo caso, la denegación del remedio del specific performance ha sido recogida por las regulaciones legales de protección de los trabajadores: así, por ejemplo, en el Derecho inglés, la s. 236 del Trade Union and Labour Relations Act 1992 (No compulsion to work) prohíbe ordenar el specific performance contra el trabajador. El specific performance del contrato de trabajo, en cambio, sí puede ser
} 
En efecto, la justificación usual para negar el cumplimiento específico, en contra de quien debe realizar el servicio, es que ello resulta demasiado invasivo de la libertad personal ${ }^{89}$. Sobre este punto, resulta interesante la lectura en clave sociológica que ofrecen SMITH у АТІYAн: "Admittedly, the aim of such orders [specifically enforce personal services or granting injunctions] is merely to get the defendant to do what he had already agreed to do. But for reasons that lie deep in the roots of the Western political tradition, orders by the state to engage in a particular job or to perform specified services -backed up, it will be remebered, by threats of imprisonment- have acquired a symbolic meaning that goes far beyond their practical significance: the carry with them the pall of servitude" ${ }^{90}$.

En cambio, cuando se trata de obtener el specific performance en contra de quien ha contratado los servicios, la justificación de la negativa es que no es razonable obligarle a seguir vinculado con una persona respecto de la cual, por la razón que sea, ha perdido la confianza ${ }^{91}$.

Por último, se estima -como una justificación para ambos casos- que el solo hecho de que el asunto haya llegado ante el tribunal es prueba de que la relación entre las partes se ha roto, y no tiene sentido obligarlas a seguir relacionándose ${ }^{92}$.

\section{Otros factores que inciden en la negativa de conceder el specific performance (máximas de equidad)}

Ya se ha dicho que lo que buscaba la Equity era ofrecer soluciones de justicia. Por esta razón, a la hora de conceder el specific performance, no solo se tenía en cuenta la naturaleza de la obligación, o la posibilidad o dificultad de obtener su cumplimiento, sino también la particular situación de los litigantes y la forma en que estos se habían comportado. Esos factores siguen siendo utilizados como criterios para justificar, en un caso concreto, la negativa al remedio del specific performance.

De esta forma, y aun cuando no parece posible ofrecer una lista exhaustiva de todos esos factores, ni resulte fácil determinar la importancia y la aplicación práctica que tiene cada uno de ellos en la actualidad ${ }^{93}$, vale la pena tener presente que un tribunal puede rechazar el

ordenado al empleador, en caso de despido injustificado, ordenando que el trabajador sea reintegrado a sus funciones [cfr. Employment Rights Act 1996 (part X)], pero, en la práctica, ello solo ocurre en la mínima parte de los casos [cfr. Treitel (2003) pp. 1029-1030].

89 Cfr., en relación con el Derecho inglés, Andrews (2011) p. 532 y CARTwright (2007) pp. 251-252; y en relación con Derecho estadounidense, FARNSWORTH (2010) pp. 185-186 y KLASS (2010) pp. 216-217.

90 Sмith y Атiяaн (2005) p. 382. En este sentido, es interesante destacar que en el caso de EE.UU., la prohibición constitucional de cualquier forma de esclavitud suele ser suficiente justificación a la negativa de ordenar el specific performance de los contratos cuyo objeto sea la prestación de servicios personales [cfr. KLASS (2010) p. 216]. La s. 1 de la $13^{\text {th }}$ amendment a la Constitución de EE.UU. dispone: "Neither slavery nor involuntary servitude, except as a punishment for crime whereof the party shall have been duly convicted, shall exist within the United States, or any place subject to their jurisdiction".

91 Cfr. Oughton y Davis (2008) p. 533.

92 Cfr. StOne (2009) pp. 635-636.

93 FRY (1911) pp. 133-549, a comienzos del siglo pasado, enumera y desarrolla veinticinco "defensas" frente a la acción de cumplimiento específico, basadas en estos criterios "equitativos". Sin embargo, no es fácil determinar la importancia y la aplicación de cada una de ellas en la actualidad, no al menos, sobre la base de la lectura de los modernos textos de Derecho contractual (como se verá inmediatamente en el cuerpo del texto): 
cumplimiento específico, en ejercicio de su poder discrecional, entre otros casos: si la contraprestación no se considera "adecuada" ${ }^{4}$; si no es posible otorgar dicho remedio también al deudor ("lack of mutuality")95; si la conducta del acreedor no lo amerita ("lack of clean hands")96; si el acreedor no está dispuesto a cumplir con su prestación ("he who seeks equity must do equity")97; si resulta demasiado gravoso o perjudicial para el deudor (hardship) ${ }^{98}$; si el acreedor, tras el incumplimiento, ha permanecido en la pasividad, y el specfic performance resulta, por ello, más perjudicial al deudor ("delay defeats equity")99; o si el cumplimiento es física o legalmente imposible (aun cuando la imposibilidad no libere al deudor) ${ }^{100}$.

\section{CONCLUSIONES}

Las principales conclusiones del estudio realizado, son las siguientes:

1. La idea generalizada de que los ordenamientos del Common Law no reconocen al acreedor un derecho al cumplimiento específico (specific performance) y que este

los autores se limitan a señalar que, además de los límites que ya han sido expuestos, existe una serie de barreras "equitativas", ofreciendo un par de ejemplos de ellas, que no siempre los mismos, así, por ejemplo, SMITH y ATIYAH (2005) p. 378; o FARNSWORTh (2010) pp. 164 y 165); si bien el tratamiento que en algunos textos se les dispensa a algunos de ellos podría ser indicio de su importancia [así, por ejemplo, STONE trata la regla de la "need of mutuality" (véase infra nota 90) y de "hardship" (véase infra nota 93), con una extensión similar a la que dispensa a la regla de la adecuación de los daños (2009) pp. 636-637). Para una exposición breve y actual de estas "máximas de equidad", que están a la base del ejercicio discrecional del tribunal, véase STOCKWELL y EDWARDS (2005) pp. 34-59.

94 La necesidad de que exista una contraprestación (consideration) es un requisito para poder obtener el cumplimiento de un contrato, pero no solo en forma específica. La defensa particular frente a la posibilidad de cumplimiento específico es que la consideration no sea adecuada, incluso en los casos en los que no justifica la anulación del contrato (sobre el punto véase por ejemplo, ANDrews (2011) p. 530; Treitel (2003) pp. 1027-1028).

95 Esto implica que, cuando el acreedor que busca el cumplimiento específico no ha cumplido aún su parte del acuerdo, la Corte debe considerar si el deudor está suficientemente protegido contra el riesgo de que el acreedor no cumpla cfr. Andrews (2011) p. 532; Stone (2009) p. 636; Treitel (2003) p. 1037. Dicho de otro modo, no se ordenará el cumplimiento específico si no es posible que el demandado obtenga, a su vez, una orden de cumplimiento específico contra el demandante. Para una exposición de esta doctrina, véase FrY (1911) pp. 231 y ss. En relación con el derecho australiano, véase CARTER et al. (2007) pp. 943-944. FARnsWOrTH (2010) p. 165, en relación con el Derecho estadounidense, sostiene que se trata de un regla que hoy está "desprestigiada".

96 La máxima aplicable es que quien recurre a la Equity, debe hacerlo con las "manos limpias" [véase STOCKWELl y EdWARds (2005) p. 43]. Se refieren a este criterio, entre otros: SMiTh y ATIYAh (2005) p. 378; ANDREWS (2011) p. 532; Stone (2009) p. 636; Treitel (2003) p. 1028. También, en relación con el Derecho estadounidense, FARNSWORTH (2010) pp. 164-165.

97 Cfr. Stockwell y Edwards (2005) pp. 42-43. A propósito de su aplicación en Australia, Carter et al. (2007) pp. 942-943, tratan este principio bajo la rúbrica "readiness and willingness of plaintiff".

98 Y también, relacionado con este motivo, porque la solución se considera injusta ("unfair") debido a que el contrato es particularmente favorable al acreedor. Sobre el punto, véase Smith y Atiyah (2005) p. 378; STONE (2009) p. 636; TReitel (2003) pp. 1026-1027; CARTER et al. (2007) p. 945.

99 "Delay may be evidence of acquiescence, so the two issues cannot be separated. A failure to bring an action may tend to confirm other slight evidence that the innocent party has accepted or agreed to the breach of contract or other ground for seeking relief, thus preventing him from enforcing his right to remedies for that breach" StockWell y EdWards (2005) p. 46. Véase, también, ANdrews (2011) p. 532; Carter et al. (2007) pp. 944-945.

100 Por ejemplo, no se puede ordenar el cumplimiento específico en contra de quien vende un terreno que no le pertenece, pero, obviamente, es responsable del incumplimiento $c f$ r. TREITEL (2003) p. 1029. 
remedio ocupa un lugar secundario frente al remedio de la indemnización de daños (damages), que se considera el remedio preferente, es una idea que tiene suficiente respaldo.

2. No obstante, y especialmente a efectos de realizar un estudio comparado con los ordenamientos de Derecho continental, dicha afirmación debe ser matizada, en consideración de los siguientes dos aspectos:

a) Por un lado, que la expresión specific performance únicamente hace referencia a la posibilidad de que el tribunal ordene directamente al deudor la ejecución de la prestación debida. Por tanto, si, desde la perspectiva de un estudio comparado, se incluyen bajo esa expresión otros casos en los puede decirse que hay "cumplimiento específico" del contrato (porque el acreedor obtiene, aunque no del deudor, la prestación in natura), entonces la afirmación sobre el lugar secundario de este remedio en el Common Law requiere, lógicamente, ser matizada.

b) Por otro lado, ha de tenerse presente que existen diversos casos en los que, en el Common Law, otorgar el remedio del specific performance se considera justificado. Y parece existir cierta tendencia a reconocer un lugar más relevante a este remedio, o al menos, a cuestionar el hecho de que este remedio deba tener un rol secundario.

3. Aun cuando el specific peformance es siempre un remedio discrecional -y por tanto, la decisión sobre la vía de protección del interés que tiene el acreedor en el cumplimiento corresponde al juez, y no al acreedor-, es posible aventurar que, en su aplicación práctica, más que hablar de un sistema jerarquizado de remedios, en los que la indemnización tenga un lugar preferente, el sistema de remedios frente al incumplimiento contractual en el Common Law puede ser descrito como un sistema diferenciado de remedios. En la exposición sintética de este esquema, es útil recurrir, por ser categorías conocidas, a la clasificación de las obligaciones según su objeto. Así:

a) Tratándose de obligaciones de no hacer, el cumplimiento específico (bajo la forma de una injunction), y sin perjuicio que puede negarse en ejercicio del poder discrecional del tribunal, en la práctica suele concederse, en palabras de TREITEL "as a matter of course" ${ }^{101}$, o sea, normalmente.

b) Tratándose de obligaciones de dar, es posible realizar una distinción según se trate de pagar una suma de dinero, de entregar un bien específico o determinado, y de entregar cosas genéricas o fungibles. Como se ha mostrado en este trabajo, la preferencia por el remedio de damages, en la práctica, solo describe adecuadamente el sistema de remedios tratándose de contratos relativos a cosas genéricas, y aún en ese caso, se advierte cierta flexibilidad en cuanto a la forma en que se determina el carácter fungible del objeto.

c) Es tratándose de obligaciones de hacer en donde la posibilidad de obtener el cumplimiento específico aparece más limitada. Por una parte, si se trata de un "servicio 
personal", simplemente se entiende que no es posible obtener el cumplimiento específico del contrato. Por otra parte, si el servicio "no es personal", la pretensión de cumplimiento específico debe, primero, superar el test de la adecuación del remedio de los damages, y además, superar otras barreras: las más importantes, el problema de la necesidad de supervisar el cumplimiento y el problema de la posibilidad de determinar con certeza que es lo que el deudor debe hacer. La aplicación de todos estos criterios sugiere la posibilidad de una subclasificación dentro de los servicios "no personales": mientras que dichas objeciones no están presentes, o resultan menos importantes, en los casos en los que la obligación consiste en fabricar y entregar un objeto -una obligación de hacer, pero que va seguida de una de dar-, dichas barreras al specific performance sí están presentes cuando la obligación del deudor consiste en una serie de actos, más o menos prolongados en el tiempo, o en los casos en los que la actividad del deudor supone el ejercicio de un mayor grado de discrecionalidad por su parte.

\section{BIBLIOGRAFÍA CITADA}

Andrews, Neil (2011): Contract Law (New York, Cambridge University Press).

Ashburner, Walter (1902): Principles of Equity (London, Butterworth and Co, 1st ed.).

BAKER, John H. (2002): An Introduction to English Legal History (Butterworths, 4a ed.).

Beale, Hugh., Fauvarque-Cosson, Bénédicte, Rutgers, Jacobien, Tallon, Denis, VogeNauer, Stefan (2010): Cases, Materials and Text on Contract Law (Oxford, Hart Publishing, $2^{\mathrm{a}}$ ed.).

BERRYMAN, Jeff (1985): "The specific performance damages continuum: An historical perspective", Ottawa Law Review, Vol. 17: pp. 295-323.

BraY, Judith (2012): A student's guide to Equity and Trust (Cambridge, Cambridge University Press).

Brown, Brendan (1974): "La equity en el derecho de los Estados Unidos de América", Boletín Mexicano de Derecho Comparado, N. 21: pp. 21-38.

Bryan, Michael, Vann, Vicki (2012): Equity and Trusts in Australia (Port Melbourne, Vic., Cambridge University Press).

Buckland, William, McNair, Arnold (1952): Roman Law and Common Law: A Comparison in Outline (Cambridge, Cambridge University Press, 2a ed.).

Burham, William (2006): Introduction to the law and legal system of the United States (St. Paul, Thompson/West, $4^{\mathrm{a}}$ ed.).

Burns, F. (1993): “The Fusion Fallacy Revisited”, Bond Law Review, Vol. 5, N. 2: pp. 151178.

Burrows, Andrew (1998): Understanding the Law of obligations (Oxford, Hart Publishing).

Burrows, Andrew (2002): "We Do This at Common Law But That In Equity", Oxford Journal of Legal Studies, Vol. 22, N. 1, pp. 1-16.

Carter, John W., Peden, Elizabeth, Tolhurst, Gregory (2007): Contract Law in Australia (Sydney, Bulterworths/Lexisnexis, 5a ed). 
Cartwright, John (2007): Contract Law. An Introduction to the English Law of Contract for the Civil Lawyer (Oxford, Hart Publishing).

Charman, Mary (2007): Contract Law (Cullompton, Willan Publishing, 4a ed.).

Cuncannon, Fionnghuala (2004): "The Case for specific performance as the primary remedy for Breach of contract in New Zealand", Victoria University of Wellington Law Review, Vol. 35: pp. 657-685.

David, René, Jauffret-Spinosi, Camille (2010): Los grandes sistemas jurídicos contemporáneos (trad. Jorge Sánchez Cordero, Universidad Nacional Autónoma de México, 11 a ed.).

De Cruz, Peter (1999): Comparative Law in a Changing World (Cavendish Publishing, 2a ed.).

Elliot, Catherine, Quinn, Frances (2009): English Legal System (Essex, Pearson/Longman, $10^{\mathrm{a}} \mathrm{ed}$.).

Esteban de la Rosa, Gloria, Gómez Valenzuela, Esperanza (2013): "La acción de cumplimiento específico en la contratación internacional” en Sánchez Lorenzo, Sixto (coord.), Derecho contractual comparado: una perspectiva europea y transnacional (Navarra, Civitas) pp. 1597-1644.

EvERs, Williamson (1977): "Towards a reformulation of the law of contracts", Journal of Libertarian Studies, Vol. 1: pp. 3-13.

FARnsworth, Edward Allan (2010): Farsworth on Contracts (Gaithersburg, MD, Aspen Publishers, $3^{\mathrm{a}}$ ed.).

Fauvarque-Cosson, Bénédicte, Mazeaud, Denis (2008): European Private Law. Materials for a Common Frame of Reference: Terminology, Guiding Principles, Model Rules (München, Sellier, 2008).

FrY, Edward (1911): A treatise on the Specific Performance of Contracts (London, Stevens and sons limited, $5^{\mathrm{a}} \mathrm{ed}$.).

Fuller, Lon; Perdue, William (1936): "The reliance interest in contract damages", The Yale Law Journal, Vol. 46: pp. 52-96 (parte 1) y pp. 373-420 (parte 2).

Furmston, Michael (2007): Cheshire, Fifoot \& Furmston's Law of Contract (Oxford, Oxford University Press, $15^{\mathrm{a}}$ ed.).

Hogg, Martin (2011): Promises and Contract Law. Comparative Perspectives (Cambridge, Cambridge University Press).

Holmes, Oliver Wendell (1882): The Common Law (Macmillan, London).

Holmes, Oliver Wendell (1998): "The Path of the Law", Boston University Law Review, Vol. 78: pp. 699-715 [publicado originalmente en 1897: Harvard Law Review, Vol. 10: pp. 457-478].

IBbETSOn, David (1997): "Fault and absolute liability in pre-modern contract law", The Journal of Legal History, Vol. 18, N. 2: pp. 1-31.

Jukier, Rosalie (2010): “Taking Specific Performance Seriously: Trumping Damages as the Presumptive Remedy for Breach of Contract", en Roach, Kent y SHarpe, Robert (edits.), Taking remedies seriously (Montreal, Canadian Institute for the Administration of Justice) pp. 85-118.

KLASS, Gregory (2010): Contract Law in the USA (Alphen aan den Rijn, Kluwer Law International). 
Kronman, Anthony T. (1978): "Specific performance", The University of Chicago Law Review, Vol. 45: pp. 351-382.

Langdell, Christopher (1908): A brief Survey of Equity Jurisdiction, (Cambridge [USA], Harvard Law review Publishing Association, 2a ed.).

McCormick, Charles (1928): "The Fusion of Law and Equity in United States Courts", North Carolina Law Review, Vol. 6: pp. 283-298.

Maitland, Frederic W. (1909): The Forms of action at Common Law. A Course of Lectures, (Cambridge, Cambridge University Press).

Maitland, F.W., Montague, F. (1915): A sketch of English Legal history (New York, G. P. Putmam's sons).

MaK, Vanessa (2008): "Specific performance in English Consumer Sales Law", en SMITS, Jan, Hass, Daniel, Hessen, Geerte (edits.) Specific performance in Contract Law: National and Other Perspectives, Antwerp-Oxford-Portland, Intersentia) pp. 121-129.

Markesinis, Basil, Unbertah, Hannes, Johnston, Angus (2006): The german law of contract. A comparative treatise (Oxford, Hart Publishing, 2a ed.).

Maxton, Julie (1993): "Some effects on the intermingling of Common Law and equity", Canterbury Law Review, Vol. 5: pp. 299-311.

Mejías Alonso, Claudia (2011): El incumplimiento resolutorio en el Código civil, (Santiago, AbeledoPerrot-LegalPublishing).

Miller, Lucinda (2009): “The Enforcement of Contractual Obligations: Comparative Observations on the Notion of Performance", en Cartwright, John, Vogenauer, Stefan, Whittaker, Simon (edits.), Reforming the French Law of Obligations. Comparative Reflections on the Avant-projet de reforme de droit des obligations et de la prescription: "the Avant-projet Catala" (Oxford, Hart Publishing) pp. 141-165.

Momberg URIBE, Rodrigo (2011): The effect of a change of circumstances on the binding force of contracts. Comparative perspectives (Antwerp, Intersentia).

Monahan, Geoff (2001): Essential Contract Law (Sydney, Cavendish Publishing, 2a ed.).

Morales Moreno, Antonio Manuel (2010): Incumplimiento del contrato y lucro cesante, Discurso leido el día 8 de febrero de 2010 en el acto de su recepción pública como Académico de Número por el Excmo. Sr. D. Antonio Manuel Morales Moreno y contestación del Excmo. Sr. D. Luis Diez-Picazo y Ponce de León (Madrid, Real Academia de Jurisprudencia y Legislación).

Morales Moreno, Antonio Manuel (2006): La modernización del Derecho de Obligaciones (Navarra, Thomson-Civitas Aranzadi).

Morineau, Marta (2004): Una introducción al Common Law (Mexico D.F., Universidad Nacional Autónoma de México).

Mulcahy, Linda (2008): Contract Law in perspective (Oxon/New York, Routledge-Cavendish, 5 ed.).

Mulheron, Rachel (2000): "New Forays of Equitable Remedies into Commercial "Personal Service” Contracts”, New Castle Law Review, Vol. 4: pp. 32-45.

Musgrave, Thomas (2009): “Comparative Contractual remedies", University of Western Australia Law Review, N. 34: pp. 300-372. 
Oughton, David, Davis, Martin (2000): Sourcebook on Contract Law (London, Cavendish Publishing, 2a ed.).

Partridge, E. (1997): Origins: A Short Etymological Dictionary of Modern English, (Oxon, Routledge, reimpresión de la $4^{\mathrm{a}} \mathrm{ed}$.).

Pérez Ragone, Álvaro, “Writ» y "Actio» en el Surgimiento y la Configuración del Proceso Civil Inglés Medieval", Revista de Estudios Histórico-Jurídicos de la Pontificia Universidad Católica de Valparaíso, N. 29: pp. 333-356.

Peтtit, Philipe (2012): Equity and the Law of Trusts (Oxford, Oxford University Press, 12a ed.).

Plucknett, Theodore F. T. (2001): A Concise History of the Common Law (New Jersey, The Lawbook Exchange, reimpresión de la $5^{\mathrm{a}}$ ed. originalmente publicada por Little, Brown and Co., Boston, 1956).

Richards, Paul (2009): Law of contract (Essex, Pearson/Longman, 9a ed.).

Romero, Louis (1986): "Specific performance of contracts in comparative law: some preliminary observations", Les Cahiers de Droit, Vol. 27: pp. 785-811.

SAmuel, Geoffrey (2001): Law of Obligations and legal remedies (London, Cavendish Publishing, $2^{\mathrm{a}}$ ed.).

Schwartz, Alan (1980): “The Case for Specific Performance”, The Yale Law Journal, Vol. 89: pp. 271-307.

Shavell, Steven (2006): "Specific Performance versus Damages for Breach of Contract: An Economic Analysis”, Texas Law Review, Vol. 84, N. 4: pp. 831-876.

Slapper, Gary (2011): How the Law works (Oxon, Routledge, 2a ed.).

SMith, Lionel (2005): "Understanding Specific Performance", en CoHEN, Nili./ McKeNDRICK, Ewan (edits.) Comparative Remedies for Breach of Contract (Oxford, Hart Publishing) pp. 221-233.

Smith, Stephen, AтіYAн, Patrick (2005): Atiyah's Introduction to the Law of Contract (Oxford, Oxford University Press, 6a ed.).

STACK, Robert (2000): "A revised remedy: Trends and Tendencies in the Law of specific performance, since Semelhago v. Paramadevan", Appeal: Review of Current Law and Law Reform, N. 6: pp. 60-73.

Stockwell, Nigel, Edwards, Richard. (2005): Trusts and Equity (Essex, Pearson/Longman, Essex, $7^{\mathrm{a}}$ ed.).

Stone, Richard (2009): Modern Contract Law (London, Cavendish Publishing, 8a ed.).

Tannenbaum, David (1954): "Enforcement of Personal Service Contracts in the Entertainment Industry”, California Law Review, Vol. 42: pp. 18-27.

Thomas, Hugh (2008): The Norman Conquest: England After William the Conqueror, (Maryland, Rowman and Littlefield Publishers).

Torsello, Marco (2006): "Remedies for breach of contract", en SMits, Jan (edit.) Elgar Encyclopedia of Comparative Law (Cheltenham, Edward Elgar Publishing) pp. 610-629.

Treitel, Guenter (1976): Remedies for a Breach of Contract. Courses of Action Open to a Party Aggrieved, en Von MEHREN, Arthur (edit.) International Encyclopedia of Comparative Law: Contracts in general [Vol. 7, cap. 16] (Tübingen/The Hague/Paris , CB Mohr-Siebeck;). 
Treitel, Guenter (2003): The Law of Contract (London, Sweet and Maxwell-Thompson, $11^{\mathrm{a}} \mathrm{ed}$.).

Turner, Chris (2007): Contract Law (London, Hodder Education, 2a ed.).

Twigg-Flesner, Christian (2008), The Europeanisation of Contract Law (London/New York, Routledge-Cavendish).

Van Caenegem, Raoul (1998): The Birth of the English Common Law (Cambrigde: Cambrigde University Press, 2a ed.).

Vidal Olivares, Álvaro (2009): "La noción de incumplimiento esencial en el Código civil", Revista de Derecho de la Pontificia Universidad Católica de Valparaíso, Vol. 32: pp. 221-258.

Von Mehren, Arthur, Murray, Peter (2007): Law in the United States (New York, Cambridge University Press).

Waterman, Thomas (1881): A practical treatise on the law relating to specific performance of contracts (New York, Baker, Voorhis \& Co.).

Watson, Alan (1990): "Roman Law and English Law: Two Patterns of Legal Development" Loyola Law Review, Vol. 36: pp. 247-268.

Willinstone, Samuel (1920): The Law of contracts (New York, Baker, Voorhis \& Co.).

Youngs, Raymond (1998): English, French and German comparative Law (London, Routledge-Cavendish).

Zimmermann, Reinhard (1992): The Law of Obligations. Roman foundations of the civilian tradition (Cape Town, Juta and Co., $1^{a}$ ed, reimpresión de 1992).

Zimmermann, Reinhard (2009): Europa y el Derecho romano (estudio introductorio y traducción de Ignacio Cremades Ugarte, Madrid, Marcial Pons).

Zweigert, Konrad, Kötz, Hein (1998): An introduction to Comparative Law (Oxford, Oxford University Press, $3^{\mathrm{a}}$ ed.).

\section{NORMAS CITADAS}

Statute of Westminster II, de 1285 (UK)

Judicature Acts 1873-1875 (UK)

The Uniform Commercial Code, 1952, (USA)

Senior Court Act 1981 (UK)

Sale of Goods Act 1979(UK)

Trade Union and Labour Relations Act 1992 (UK)

Employment Rights Act 1996 (UK)

Directiva 1999/44/CE del Parlamento Europeo y del Consejo, de 25 de mayo de 1999, sobre determinados aspectos de la venta y las garantías de los bienes de consumo (Unión Europea).

\section{JURISPRUDENCIA CITADA}

Pusey v. Pusey (1684) 23 Eng. Rep. 465.

Nutbrown v. Thornton (1804) 10 Ves. 159. 
Lumley v. Wagner (1852) EWHC Ch J96.

Falcke v. Gray (1859) 62 Eng. Rep. 250.

Warner Bros v. Nelson (1937) 1 KB 209.

Beswick v. Beswick (1968) UKHL 2.

Sky Petroleum Ltd v. VIP Petroleum Ltd (1974) 1 WLR 576.

Societé des Industries Metallurgiques SA v. Bronx Engineering Co. Ltd (1975) 1 Lloyd's Rep 465 CA.

United Scientific Holdings Ltd v. Burnley Borough Council (1978) AC 904.

Perry \& Co v. British Railways Board (1980) 2 All ER 579.

Semelhago v. Paramadevan (1996) 2 S.C.R. 415.

Co-operative Insurance Society Ltd v. Argyll Stores (1997) UKHL 17.

Attorney General v. Blake and Another (2000) UKHL 45. 
